\title{
Transcriptome analysis of Klf15-mediated inhibitory functions in a mouse deep venous thrombosis model
}

\author{
JIN ZHOU*, XUELING ZHAO* , SHIWEI XIE and RUDAN ZHOU \\ Department of Orthopedics, The First Affiliated Hospital of Kunming Medical University, \\ Kunming, Yunnan 650032, P.R. China
}

Received October 23, 2019; Accepted February 24, 2020

DOI: $10.3892 /$ ijmm.2020.4538

\begin{abstract}
Krüppel-like family (KLF) members are important regulators of proinflammatory activation in the vasculature. A transcriptome study involving RNA sequencing (RNA-seq) and quantitative PCR (qPCR) was performed to investigate Klf15 and Klf15-regulated gene levels in C57BL/6 mice with inferior vena cava thrombi and in control (Blank) mice. A total of 2,206 differentially expressed genes (DEGs), including 1,330 upregulated and 876 downregulated genes, were identified between the deep venous thrombosis (DVT) group and the Blank group. Additionally, 1,041 DEGs (235 upregulated and 806 downregulated) were identified between the Klf15-small interfering RNA (siRNA) and Klf15-negative control (NC) groups. The DEGs were subjected to Gene Ontology and Kyoto Encyclopedia of Genes and Genomes enrichment analyses, and qPCR was conducted to validate the results. A total of seven significant DEGs were selected from the RNA-seq results. Matrix metalloproteinases (Mmp)12, Mmp13, Mmp19, Arg1, Ccl2, heme oxygenase-1 and Fmo3 levels were significantly higher, while Klf15 levels were lower, in the DVT group than in the Blank group. Fmo3 and Mmp19 have not been previously identified as DVT-associated DEGs. Klf15, Mmp12 and Mmp13 levels were compared between the Klf15-siRNA and Klf15-NC groups. Mmp12 and Mmp13 expression was significantly higher, while that of Klf15 was lower, in the Klf15-siRNA group than in the Klf15-NC group. Critical roles of Klf15, Mmp12 and Mmp13 have been identified, which have not previously been shown to help regulate DVT initiation and progression. Moreover, Klf15-mediated regulation of DVT may be modulated by downregulation of various genes, such
\end{abstract}

Correspondence to: Ms. Rudan Zhou, Department of Orthopedics, The First Affiliated Hospital of Kunming Medical University, 295 Xichang Road, Wuhua, Kunming, Yunnan 650032, P.R. China E-mail: kittypku@qq.com

\section{*Contributed equally}

Key words: deep venous thrombosis, RNA-sequencing, Krüppel-like family 15 , matrix metalloproteinases as Mmp12 and Mmp13, potentially providing a theoretical foundation and diagnostic criteria for DVT treatment.

\section{Introduction}

Deep venous thrombosis (DVT) is one of the most common vascular diseases and is associated with high mortality and complex therapeutic processes (1). Thrombolytics and interventional therapies are still the mainstream treatments for DVT, but they are limited by a low cure rate and a high postoperative recurrence rate (2). The current therapeutic methods are restricted, especially regarding the prolonged time for diagnosis and treatment (3). Considering the complex mechanisms and various regulatory factors of DVT, studies on DVT have focused on the underlying regulatory genes, providing a valuable foundation for the diagnosis and treatment of DVT (4-6).

Krüppel-like factor 15 (Klf15), a transcriptional regulatory factor, is involved in various pathophysiological processes, such as cell differentiation, apoptosis and tumor formation, which are closely related to cardiovascular diseases such as hypertension, atherosclerosis and coronary heart disease (4). Klf15 is widely expressed in tissues and organs, especially in the heart, liver, kidneys and skeletal muscles (7). Lu et al (8) reported that similarly to other members of the KLF family, Klf15 inhibits NF- $\mathrm{kB}$ activation in vascular smooth muscle by interacting with p300 (Klf15-p300), thereby inhibiting downstream target genes and inflammatory responses. Moreover, the expression level of Klf15 significantly decreased in mouse aortic smooth muscle cells treated with the oxidized component POVPC and human atherosclerotic tissues, which revealed that Klf15 plays a key role in the formation of atherosclerosis $(7,8)$. Studies revealing the relationship and the genetic interaction between DVT and Klf15 are urgently needed. Therefore, transcriptome analysis of Klf15 in a mouse inferior vena cava (IVC) thrombosis model was performed to identify the functions of Klf15 and its relationship with the regulatory process and formation of DVT.

High-throughput sequencing, or next-generation sequencing, is a novel genomic research technique characterized by high data output and involves RNA sequencing (RNA-seq); high-throughput sequencing can be utilized in the analysis of various transcriptional and functional regions (9). Strikingly, extensive data resources can be provided via 
high-throughput sequencing to enable the identification and screening of target genes or differentially expressed genes (DEGs) in the whole genome, which is important for analyzing the regulatory relationships between genes and disease pathogenesis (10).

Previous studies have investigated the role of Klf15 in atherosclerosis (8) and vascular smooth muscle cells (VSMCs) (11). Klf15 is a regulator of VSMC proinflammatory activation and overexpression of Klf15 can protect vascular endothelial cells against dysfunction (12). Although the pathogeneses of atherosclerosis and DVT are different, endothelial cells are important for both atherosclerosis and DVT. Disruption of the endothelium and the release of plaque constituents into the lumen of the blood vessel can trigger arterial thrombosis $(13,14)$. Abnormal blood flow, altered properties of the blood itself and changes in the endothelium can trigger venous thrombosis. In contrast to what is observed in atherosclerosis, venous endothelial cells remain intact, but their dysfunction can trigger DVT (15). According to our knowledge, no reports have studied Klf15 in DVT. The research on Klf15 in atherosclerosis prompted the present study to hypothesize that Klf15 can protect against DVT by affecting venous endothelial cells. Preliminary experiments were performed in C57/BL/6 mice and the results showed that inhibition of Klf15 induced DVT. In this study, the regulatory relationship and genetic interactions between DVT and Klf15 were investigated, revealing a new regulatory mechanism in a mouse model that could contribute to the diagnosis and treatment of DVT.

\section{Materials and methods}

Mouse and animal studies. The current study was performed with 40 C57BL/6 female mice (age, 8-10 weeks; weight, $20 \pm 3 \mathrm{~g}$ ) that were purchased from the SPF animal laboratory of Kunming Medical University (Kunming, China). The mice were divided into four groups $(\mathrm{n}=10)$, according to a random grouping design. Then, the mice were fed at the experimental center of the SPF animal laboratory at Kunming Medical University with free access to food and water, a constant temperature of $21-25^{\circ} \mathrm{C}$, a humidity level at $50-65 \%$, under a 12-h light/dark cycle with proper ventilation. Next, a 2-3 week feeding period was conducted until the mice reached $\geq 25 \mathrm{~g}$ per mouse. The mice were observed twice daily to monitor their health and behavior. All animal experiments were performed following approval from the Animal Experiment and Ethics Committee of Kunming Medical University.

Generation of IVC thrombus in C57BL/6 mice. Once the weight of the mice exceeded $25 \mathrm{~g}$, modeling of IVC thrombi in C57BL/6 mice was performed in each mouse except the Blank group. Mice were separated into four groups: The Blank group, the DVT group, the Klf15-NC group and the Klf15-small interfering (si)RNA group. The Blank and DVT groups were first generated, and mice in the DVT group underwent an operation to generate an IVC thrombus by utilizing a string to induce artificial stenosis of the IVC for thrombus formation (16). IVC thrombosis in mice was first modeled. After $24 \mathrm{~h}$, the thrombi were acquired. During the perioperative period, the mice were monitored twice daily and they did not appear to be in distress or to exhibit obvious behavioral abnormalities. After the IVC thrombi were collected for further investigation, no other procedures were performed on the mice. Isoflurane was used as the inhaled agent to produce general anesthesia in mice. During the perioperative period of the experiment, the inhalant anesthetic isoflurane was utilized to induce and maintain general anesthesia to minimize animal pain and suffering and limit the discomfort that can accompany scientific research. Isoflurane was first used at $2 \%$ for induction and then at $1-1.5 \%$ for maintenance. Mice that have undergone IVC removal are likely to experience great pain and distress; thus, euthanasia was considered as the humane option. Euthanasia was conducted $24 \mathrm{~h}$ after the IVC thrombus operation. The mice were first anesthetized with $5 \%$ isoflurane until they stopped moving or appeared to be unconscious. Next, cervical dislocation was conducted, separating the cervical vertebrae from the skulls of the mice. An array of criteria was used to confirm the success of euthanasia, including arrest of pulse and breathing, lack of corneal reflex and inaudibility of respiratory sounds and heartbeat sounds upon examination with a stethoscope. The same process was performed for the IVC of the Klf15-NC and Klf15-siRNA groups, and there was an additional caudal vein injection with $0.9 \%$ normal saline (NS) in the Klf15-NC group and with Klf15 siRNA: 5'-CCTGTGAAG GAGGAACATT-3' (Guangzhou RiboBio Co., Ltd; 10 nmol per mouse) in the Klf15-siRNA group, which was performed $24 \mathrm{~h}$ before the operation. A total of $40 \mathrm{C} 57 \mathrm{BL} / 6$ mice were used in the present experiment, 36 of which were euthanized by cervical dislocation under anesthesia; four died due to hemorrhagic shock. The success of DVT modeling was judged by direct observations of the weights of the thrombi and vessels collected from the mice. In the present experiments, when $7-8 \mathrm{~mm}$ thrombi or vessels from mice were examined, most of the thrombi weighed $>10 \mathrm{mg}$ and most of the vessels weighed $<10 \mathrm{mg}$.

RNA isolation and RNA-seq. On the basis of morphological experiments, thrombi in the IVC of mice and the vessels themselves were collected for examination. According to the manufacturer's protocol, RNA was extracted with TRIzol Reagent at $4^{\circ} \mathrm{C}$. RNA purity was determined using a NanoPhotometer spectrophotometer (IMPLEN) and the concentration was measured using a Qubit RNA Assay kit in a Qubit $^{\circledR}$ 2.0 Fluorometer (Thermo Fisher Scientific, Inc.). RNA integrity was assessed using the RNA Nano 6000 Assay kit of the Bioanalyzer 2100 system (Agilent Technologies, Inc.). Then, RNA degradation and contamination were monitored on $1 \%$ agarose gels. Furthermore, RNA purity was assessed using the RNA Nano 6000 Assay kit of the Bioanalyzer 2100 system (Agilent Technologies, Inc.). Thus, qualified RNA was used as a material for later analyses and provided samples for RNA-seq.

RNA-seq data and bioinformatics analysis. High-throughput sequencing was used to obtain and identify raw reads in samples. Further evaluation of the quality of the clean reads was performed to discard low-quality reads, which had either $>50 \%$ of bases with a $Q$ value $\leq 20$ or $>5 \%$ unrecognized sequences ('N'). After obtaining the high-quality clean reads, the reads were mapped to the human reference genome to enable downstream gene analysis. 

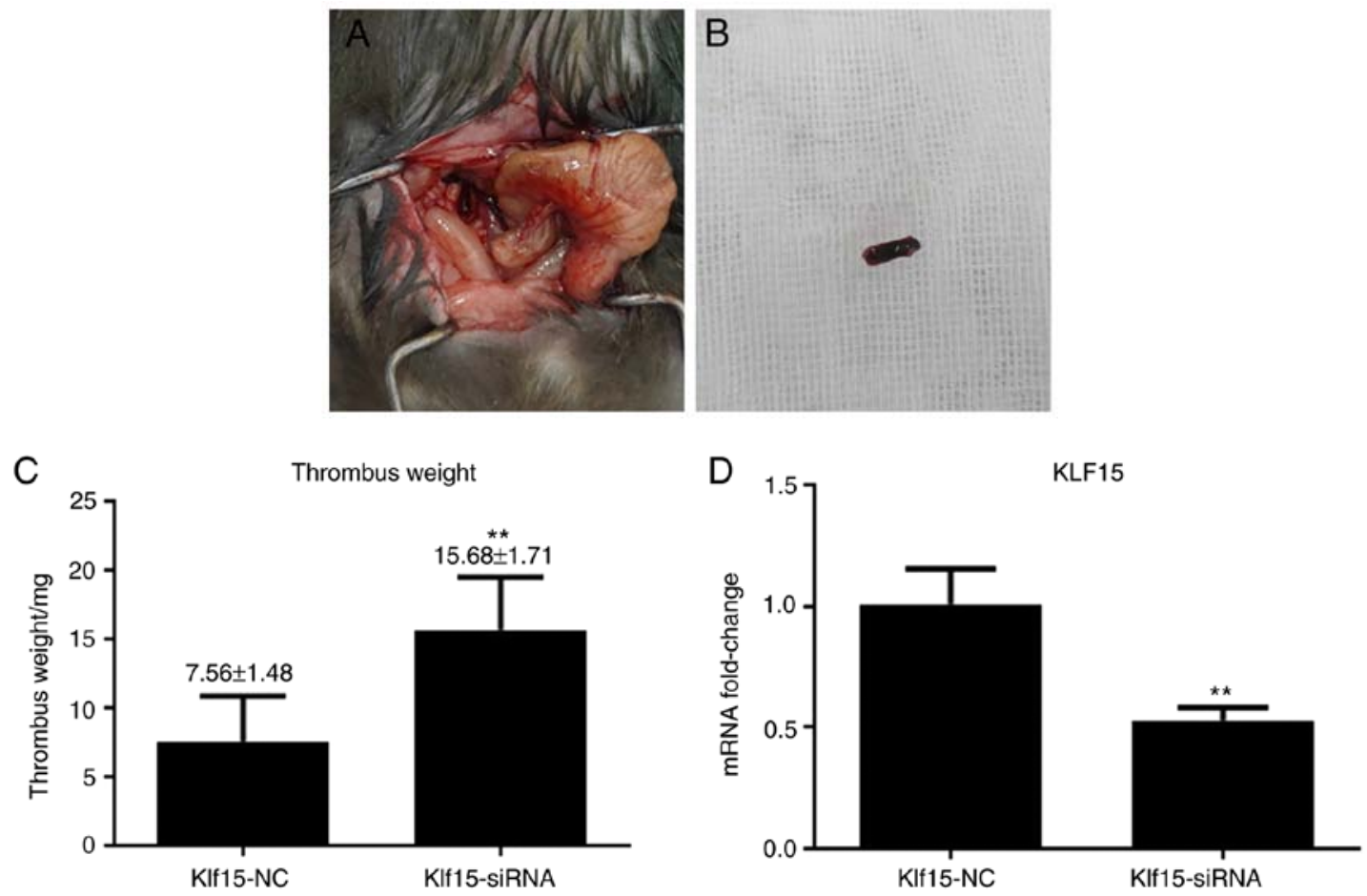

Figure 1. Modeling of DVT mice and weighing of the thrombi. (A) Inferior vena cava thrombosis. (B) Thrombi in mice with successful DVT modeling. (C) Wet weights of the mouse thrombi. After establishing a mouse inferior vena cava thrombosis model, the thrombi and vessel walls were removed from the mice in the two groups. The weights of the thrombi in the Klf15-siRNA group were significantly higher than those in the Klf15-NC group **P $<0.01$. (D) Quantitative PCR analysis of the expression of Klf15 in the two groups. The expression of Klf15 in the Klf15-siRNA group was significantly lower than that in the Klf15-NC group. ${ }^{* *} \mathrm{P}<0.01$ Klf15-NC. si, small interfering; Klf15, Krüppel-like family 15; NC, negative control; DVT, deep venous thrombosis.

For analysis of the expression levels of transcripts and the correlation of replicates, the fragments per kilobases per million mapped reads (FPKM) method was utilized in Pearson's correlation analysis to identify DEGs among each group of transcript sequences, which was determined by genetic length and the reads mapped to the human reference genome.

To detect the DEGs among the groups, DESeq2 was used, which provides statistical routines for determining differential expression in digital gene expression data using a model based on a negative binomial distribution. The resulting P-values were adjusted using Benjamini and Hochberg's approach for controlling the false discovery rate. Genes with an adjusted $\mathrm{P}<0.05$ according to DESeq2 were considered differentially expressed.

GO enrichment analysis of DEGs was implemented by the clusterProfiler R package 3.14.3 (17), in which the gene length bias was corrected. GO terms with corrected $\mathrm{P}<0.05$ were considered significantly enriched with DEGs. KEGG is a database resource used to elucidate the high-level functions and utilities of biological systems, such as the cell, organism and ecosystem, from molecular-level information, especially large-scale molecular datasets generated by genome sequencing and other high-throughput experimental technologies (http://www.genome.jp/kegg/). The clusterProfiler $\mathrm{R}$ package was used to test the statistical enrichment of DEGs in KEGG pathways.

qPCR analysis of DEGs. For validation of the expression of genes and the consistency of these two comparisons, qPCR analysis of DEGs was conducted according to the manufacturer's protocol for Maxima ${ }^{\circledR}$ SYBR-Green/ROX qPCR Master Mix (2X) (MBI Fermentas; Thermo Fisher Scientific, Inc.) on an ABI PRISM ${ }^{\circledR} 7300 \mathrm{HT}$ system (Applied Biosystems; Thermo Fisher Scientific, Inc.). The thermocycling conditions were as follows: Initial denaturation at $95^{\circ} \mathrm{C}$ for $10 \mathrm{~min}(1 \mathrm{cycle})$, followed by denaturation at $95^{\circ} \mathrm{C}$ for $15 \mathrm{sec}$ and annealing and extension at $60^{\circ} \mathrm{C}$ for $60 \mathrm{sec}(40$ cycles). The primer sequences were obtained using the $2^{-\Delta \Delta C q}$ method (8) [ABI DataAssist ${ }^{\mathrm{TM}}$ v3.0 software (Thermo Fisher Scientific, Inc.)] and were as follows: KLF15 (77 bp) forward: 5'-CTT CCCTGAATTTCTGTC-3' and reverse: 5'-ATTCTTCAA TCTCCTCCA-3'; Mmp12 (88 bp) forward: 5'-CAGCATTCC AATAATCCAA-3' and reverse: 5'-GTATGTCATCAGCAG AGA-3'; Mmp13 (79 bp) forward: 5'-GTGATGATGATGATG ATGAC-3' and reverse: 5'-GCAGGATGGTAGTATGATT-3'; Arg1 (71 bp) forward: 5'-AACACGGCAGTGGCTTTA-3' and reverse: 5'-TCAGTCCCTGGCTTATGG-3'; Ccl2 (120 bp) forward: 5'-TGGGTCCAGACATACATT-3' and reverse: 5'-ACGGGTCAACTTCACATT-3'; Fmo23 (88 bp) forward: 5'-GAGTCTGGGACGATGGCTAC-3' and reverse: 5'-GAG ATGGCGGTGGGTAAG-3'; heme oxygenase-1 (Hmox1) (88 bp) forward: 5'-TCACAGATGGCGTCACTT-3', and reverse: 5'-AGCGGTGTCTGGGATGAG-3'; Mmp19 (113 bp) forward: 5'-GATGCTGCCGTTTACTCT-3' and reverse: 5'-GGTTGGGCTCTACTCTGTT-3'; $\beta$-actin (87 bp) forward: 5'-TATGGAATCCTGTGGCATC-3' and reverse: 5'-GTGTTG GCATAGAGGTCTT-3'.

Statistical analysis. Prism 7 (GraphPad Software, Inc.) was used for all statistical analyses. The results from mouse 
thrombi (Figs. 1 and 6) are presented as the mean \pm SEM. The experiments were repeated three times. An unpaired two-tailed Student's t-test between two groups was used for statistical significance of differences analyzed. $\mathrm{P}<0.05$ was considered to indicate a statistically significant difference.

\section{Results}

Effect of Klf15 on thrombosis formation and the wet weight of the mouse thrombus. Klf15 has been shown to be critical for the initiation and progression of vascular inflammation (6). In this study, to identify the effects of Klf15 on DVT, morphological experiments were conducted on mice with IVC thrombi, which were divided into four groups: The Blank group, the DVT group, the Klf15-NC group with $0.9 \%$ NS caudal vein injection and the Klf15-siRNA group with Klf15-siRNA caudal vein injection (Table I). The vessels were removed from the mice of Blank group. If there was a blood clot blocking the vein based on direct observations (Fig. 1A), the clot was removed from the mouse for further examination (Fig. 1B). The results revealed that the weight of the thrombus in the Klf15-siRNA group was increased compared with that in the Klf15-NC group. As shown in Fig. 1C, compared with the Klf15-NC group, the Klf15-siRNA group with Klf15-siRNA injection (and thus with significantly reduced Klf15 expression) exhibited a significantly increased thrombus wet weight (Fig. 1D). Thus, Klf15 is significantly associated with thrombus formation and weight.

RNA-seq results, data quality assessment and mapping. After performing the morphological experiments previously described, genetic analysis was conducted by RNA-seq, a method of high-throughput sequencing, to investigate transcriptional gene abundance and simultaneously study active regions of transcription.

Samples were divided into four groups: The Blank group, the DVT group, the Klf15-NC group (0.9\% NS caudal vein injection) and the Klf15-siRNA group (Klf15-siRNA caudal vein injection). A total of $\sim 25.8$ and 28.1 million raw reads were collected from the DVT and Blank groups, respectively, while 29.6 and 30.3 million raw reads were obtained from the Klf15-NC and Klf15-siRNA groups, respectively. Then, further analysis was performed to obtain high-quality clean reads and the low-quality reads, which had either $>50 \%$ of bases with a $\mathrm{Q}$ value $\leq 20$ or $>5 \%$ of unrecognized sequences ('N'), were discarded. Consequently, $\sim 24.8$ and 26.8 million high-quality clean reads were obtained from the DVT and Blank groups, respectively, 29.1 and 29.6 million high-quality clean reads were obtained from the Klf15-NC and Klf15-siRNA groups, respectively. Then, the clean reads were mapped to the human reference genome for downstream gene analyses. As a result, the rates of mapping for the DVT group and the Blank group were 89.40 and $90.22 \%$, respectively, while the rates of mapping for the Klf15-NC group and the Klf15-siRNA group were 89.26 and $90.12 \%$, respectively, which demonstrated the quality of the gene mapping. The results of the RNA-seq reads are listed in Table II and the mapping results are listed in Table III. The high-quality reads for different groups were collected for further analyses.
Correlation analysis, principal component analysis (PCA) and clustering analysis. To study the correlation and clustering of the transcript sequences, the FPKM method was used to evaluate the expression level of transcripts and the correlations between replicates, which were determined in each sample by utilizing the genetic length and the reads mapped to the human reference genome.

As shown in Fig. 2A and B, except for the Blank 1 group with an $\mathrm{R}^{2} \leq 0.8$, Pearson's correlation analysis indicated that the distribution of the FPKM values were significantly consistent for all replicates compared with each group $\left(R^{2} \geq 0.8\right)$.

Furthermore, PCA was performed to evaluate the differences among groups and the consistency of samples within groups, which were expected to be distant from each other in different groups and clustered within the same group. The Blank 1 group, the DVT 1 group and the Klf15-siRNA 3 group were discarded, as these groups exhibited poor correlations with their own groups. Thus, clustering analysis was performed on groups of samples that were further separated into subgroups according to the PCA results: The Blank group (Blank 2 and Blank 3), the DVT group (DVT 2 and DVT 3), the Klf15-siRNA group (Klf15-siRNA2 and Klf15-siRNA5) and the Klf15-NC group (NC1, NC3 and NC4). The clustering analysis showed repeatable and correlative characteristics in the data. The results of the clustering analysis demonstrated gene expression differences between the Blank group and the DVT group and between the Klf15-siRNA group and the Klf15-NC group (Fig. 2C and D).

Identification of DEGs. For analysis of gene expression and the DEGs, data processing was conducted with the essential conditions of an adjusted $\mathrm{P}<0.05$ and $\log 2$-fold-change (FC) for the determination of gene regulation, which was $\log 2 \mathrm{FC}>1$ for upregulated genes and $\log 2 \mathrm{FC}<-1$ for downregulated genes. In total, 2,206 DEGs were identified from the comparison of the DVT group and Blank group, including 1,330 upregulated genes and 876 downregulated genes, which are represented as red points and green points in the volcano plot, respectively (Fig. 3A). Gene expression analysis also revealed 1,041 DEGs between the Klf15-siRNA group and the Klf15-NC group, with 235 upregulated genes and 806 downregulated genes (Fig. 3B). The number of DEGs in each comparison is listed in Table IV. As shown in Table $\mathrm{V}$, the expression levels of these genes showed significant differences in the DVT group compared with in the Blank group and the genes mainly clustered into three gene families; there were five genes in the Mmp family, four genes in the IL family, and 13 genes in the chemokine family. The expression levels of Mmp3, Mmp8, Mmp9, Mmp13 and Mmp19 in the DVT group were significantly increased compared with those in the Blank group. The expression of Mmp12 was also increased in the DVT group when compared with the Blank group; however, this finding was not significant. Genes in the interleukin (IL) family, including Il-lr2, Il-la, Il-lb and Il-6, were expressed more highly in the DVT group than in the Blank group. Moreover, the expression levels of genes in the $\mathrm{Cc}$ and $\mathrm{Cx}$ families of the DVT group were higher than those of the Blank group, except the expression level of Cc121a, which was lower in the DVT group than in the Blank group. 
A

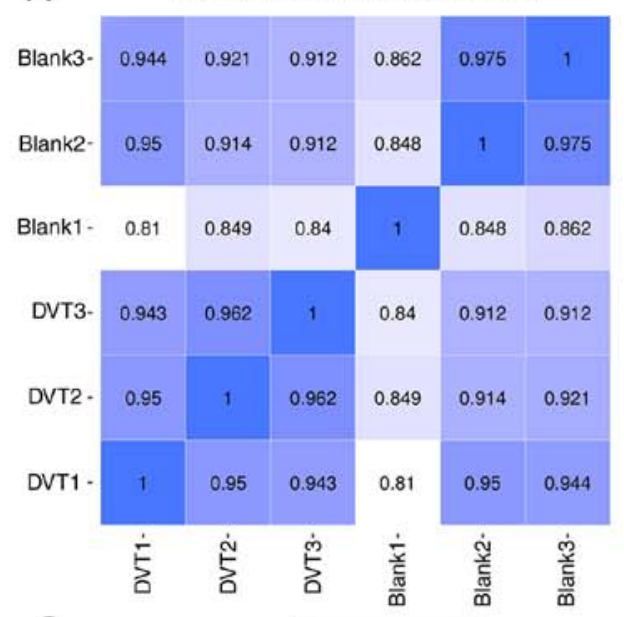

C

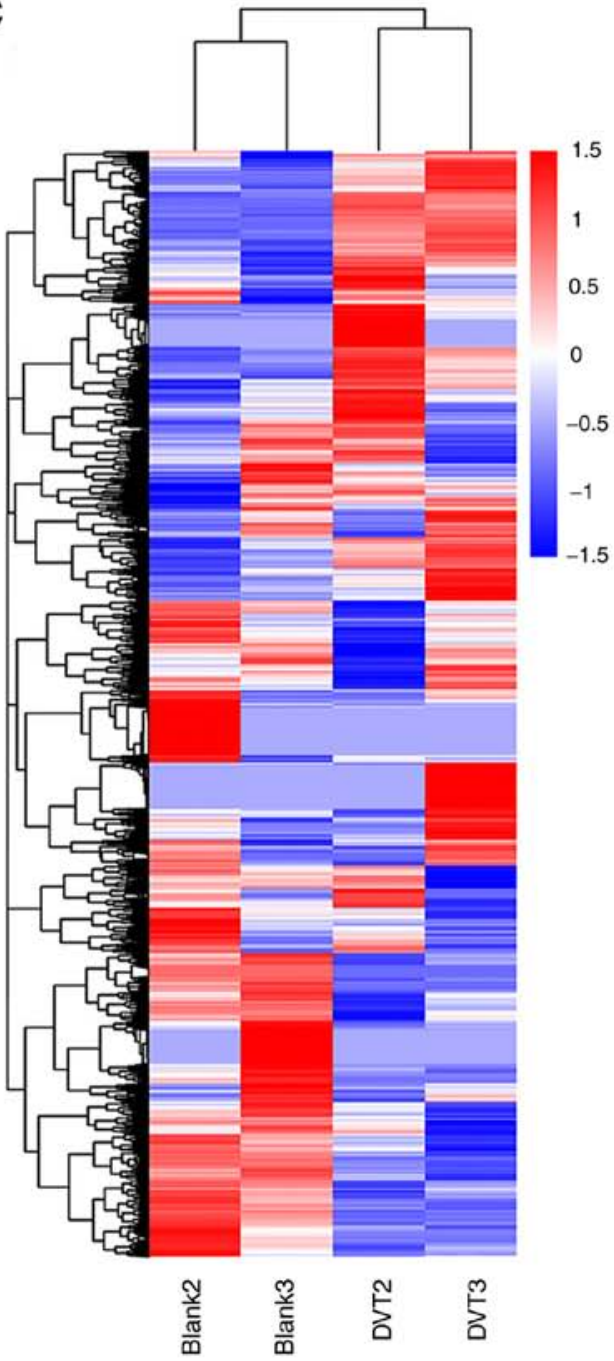

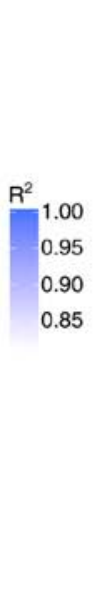

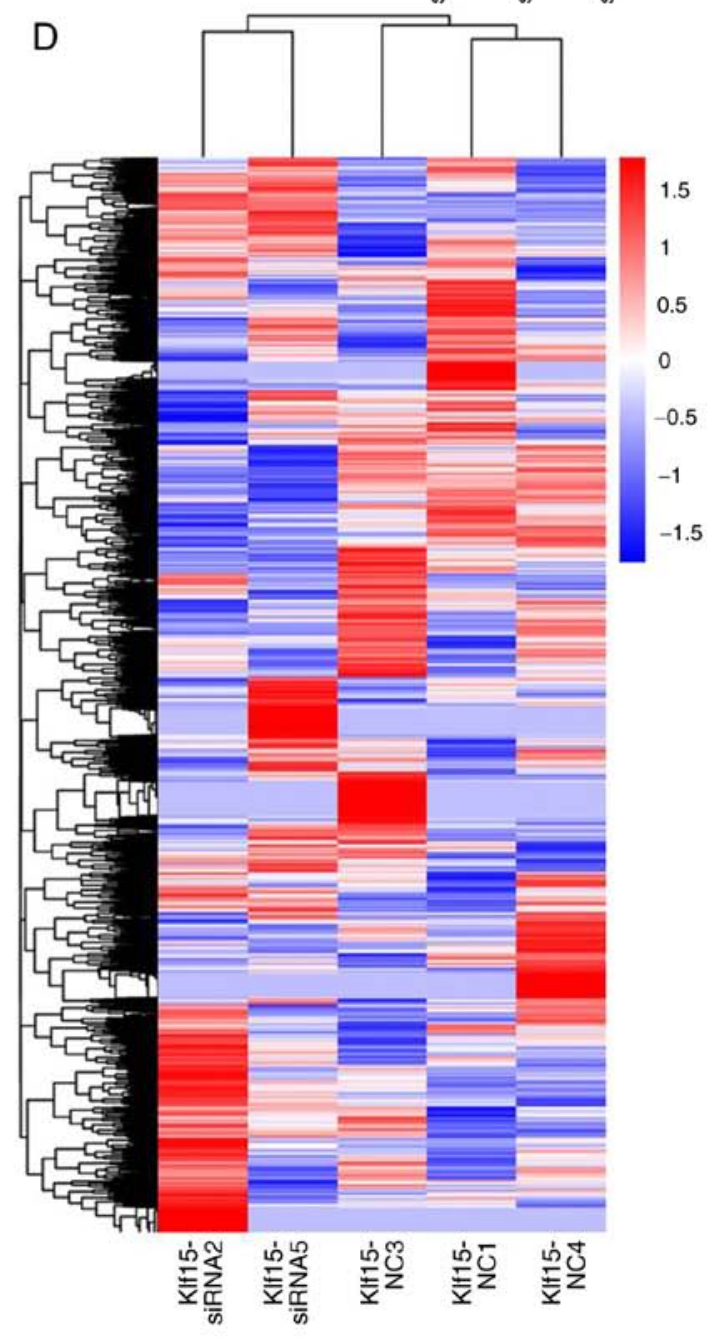

B

Pearson correlation between samples

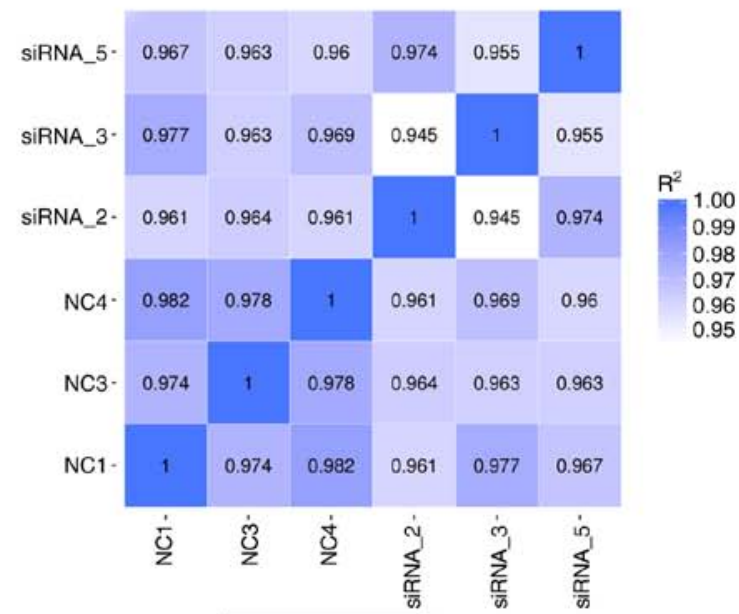

Figure 2. Correlation and clustering analyses for the comparison of the DVT group and the Blank group, and the comparison of the Klf15-NC group and the Klf15-siRNA group. Due to poor correlation according to Pearson's analysis, the Blank 1 group, DVT 1 group and Klf15-siRNA 3 group were excluded from further analyses. (A) Correlation heat map for the Blank group and DVT group. The essential threshold for further analysis was a Pearson's correlation $\mathrm{R}^{2} \geq 0.8$ and the results indicated that the distribution of the fragments per kilobases per million mapped reads values were consistent for all replicates in each group $\left(\mathrm{R}^{2} \geq 0.8\right)$, except in the Blank 1 group. (B) Correlation heat map for the Klf15-NC and Klf15-siRNA groups. The results for the Klf15-siRNA 3 group revealed significant consistency in all replicates. (C) The clustering analysis demonstrated gene expression differences between the Blank group and DVT group. (D) The clustering analysis also demonstrated gene expression differences between the Klf15-NC group and the Klf15-siRNA group. si, small interfering; Klf15, Krüppel-like family 15; NC, negative control; DVT, deep venous thrombosis.

As shown in Table V, there were four DEGs (Selp, Plaur, Hmoxl and Argl) that were detected from the comparison between the DVT group and the Blank group, which correlated with the previously obtained results. 
A

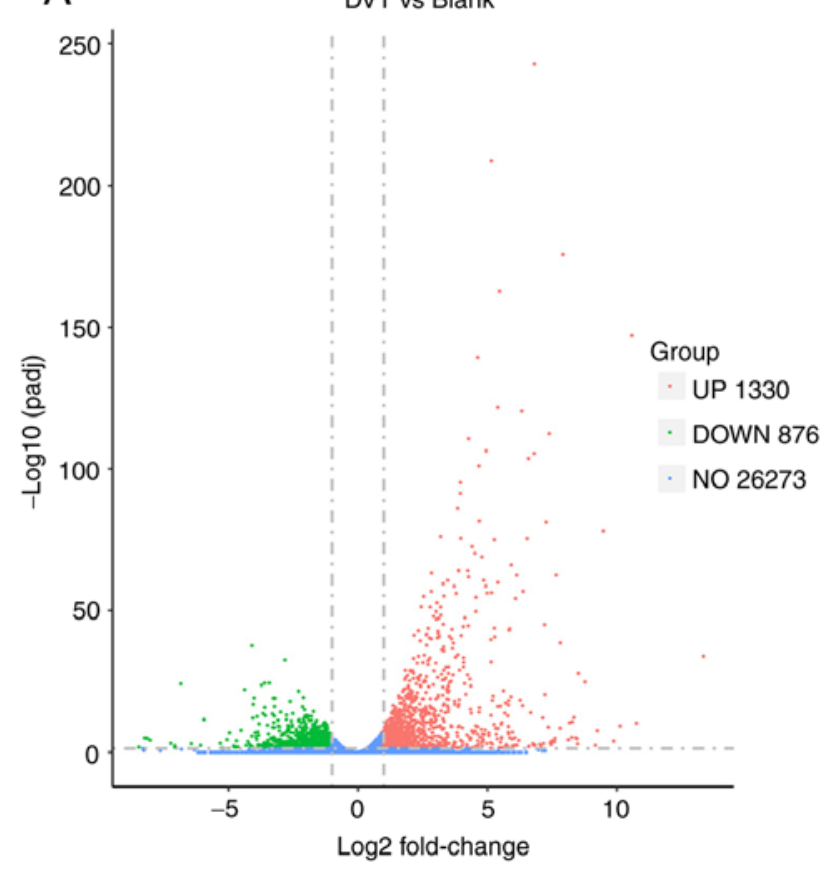

B

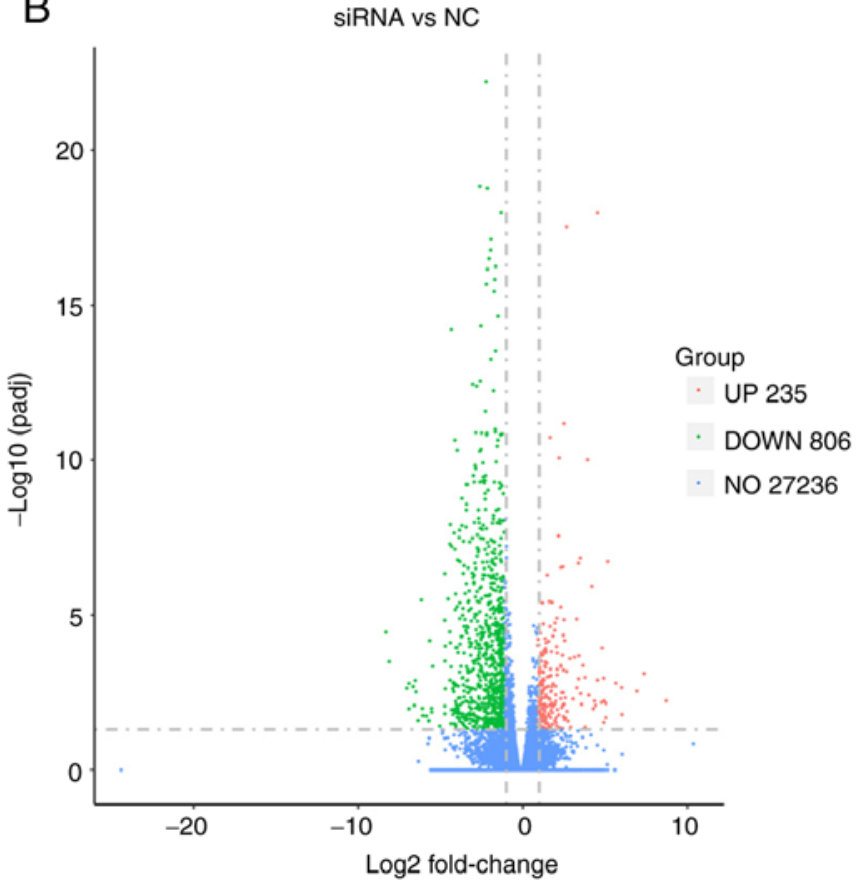

Figure 3. Volcano plot of differentially expressed genes. Each point represents one gene that is detectable in both groups. The red points represent the significantly upregulated genes; the green points represent the significantly downregulated genes; the blue points represent genes that are expressed without a significant difference. (A) Volcano plot between the Blank group and the DVT group. (B) Volcano plot between the Klf15-NC group and the Klf15-siRNA group. si, small interfering; Klf15, Krüppel-like family 15; NC, negative control; DVT, deep venous thrombosis.

Notably, two DEGs were identified, Fmo3 and Mmp19, that had not been previously detected in DVT and are listed in Table V.

As shown in Table VI, three genes, Klf15, Mmp12 and Mmp13, showed higher expression in the Klf15-siRNA group than in the Klf15-NC group, except for the level of Klf15 itself due to the caudle vein injection of Klf15 siRNA in mice of the Klf15-siRNA group.

GO analysis of DEGs. Previously in this study, DEGs were identified among different groups. Thus, GO enrichment analysis was performed to discover the biological processes of these DEGs, which demonstrated significant functions of gene expression in different groups. Then, the GO terms were divided into three categories: Biological process (BP), cellular component (CC) and molecular function (MF).

As shown in Fig. 4A and B, the top 30 ranked GO terms of the comparison between the DVT group and the Blank group were selected for the bar graph and scatter plot. Consequently, 'leukocyte migration' was the most significantly enriched term. Then, 'positive regulation of locomotion', 'positive regulation of cell motility' and 'positive regulation of cell migration' accounted for the most enriched terms in the BP category. In addition, in Fig. 4C and D, the top 30 ranked GO terms from the comparison of the Klf15-siRNA group and the Klf15-NC group showed that 'axon, postsynapse' and 'presynapse' were the most highly enriched terms. At the same time, 'the regulation of ion transmembrane transport', 'signal release' and 'modulation of synaptic transmission' were abundant in both the CC category and the MF category.

The results of the GO analysis revealed the distribution of genes in different biological functions, from which information regarding DEGs that may be beneficial to further study could be obtained.

KEGG pathway analysis of DEGs. To characterize the coordinative relations between genes and the roles of genes in biological functions, DEGs were analyzed by KEGG enrichment analysis, in which biochemical metabolic and signal transduction pathways were detected from the included DEGs.

The results in Table VII revealed that 50 pathways with significant expression $(\mathrm{P}<0.01)$ were identified in the comparison between the DVT group and the Blank group. In Fig. 5A and B, the top 20 ranked pathways are listed in the bar graph and bubble diagram; numerous signal transduction pathways were notably enriched, including the 'HIF-1 signaling pathway', 'Th17 cell differentiation', 'the intestinal immune network for IgA production', 'TNF signaling pathway', 'cell adhesion molecules (CAMs)', 'the PI3K-Akt signaling pathway, 'ECM-receptor interactions', 'the Jak-STAT signaling pathway' and 'the IL-17 signaling pathway'. Moreover, BP terms, including 'the cytokine-cytokine receptor interaction', 'hematopoietic cell lineage', 'Th17 cell differentiation', 'CAM', 'ECM-receptor interaction', 'glycolysis/gluconeogenesis', 'osteoclast differentiation', 'Staphylococcus aureus infection', 'Th1 and Th2 cell differentiation', and 'complement and coagulation cascade terms', were significantly enriched in the analysis.

In the comparison between the Klf15-siRNA group and the Klf15-NC group, 23 pathways with significant expression $(\mathrm{P}<0.01)$ were identified and are listed in Table VIII. The top 20 ranked pathways are shown in Fig. 5C and D. The results revealed that several signal transduction pathways 
A

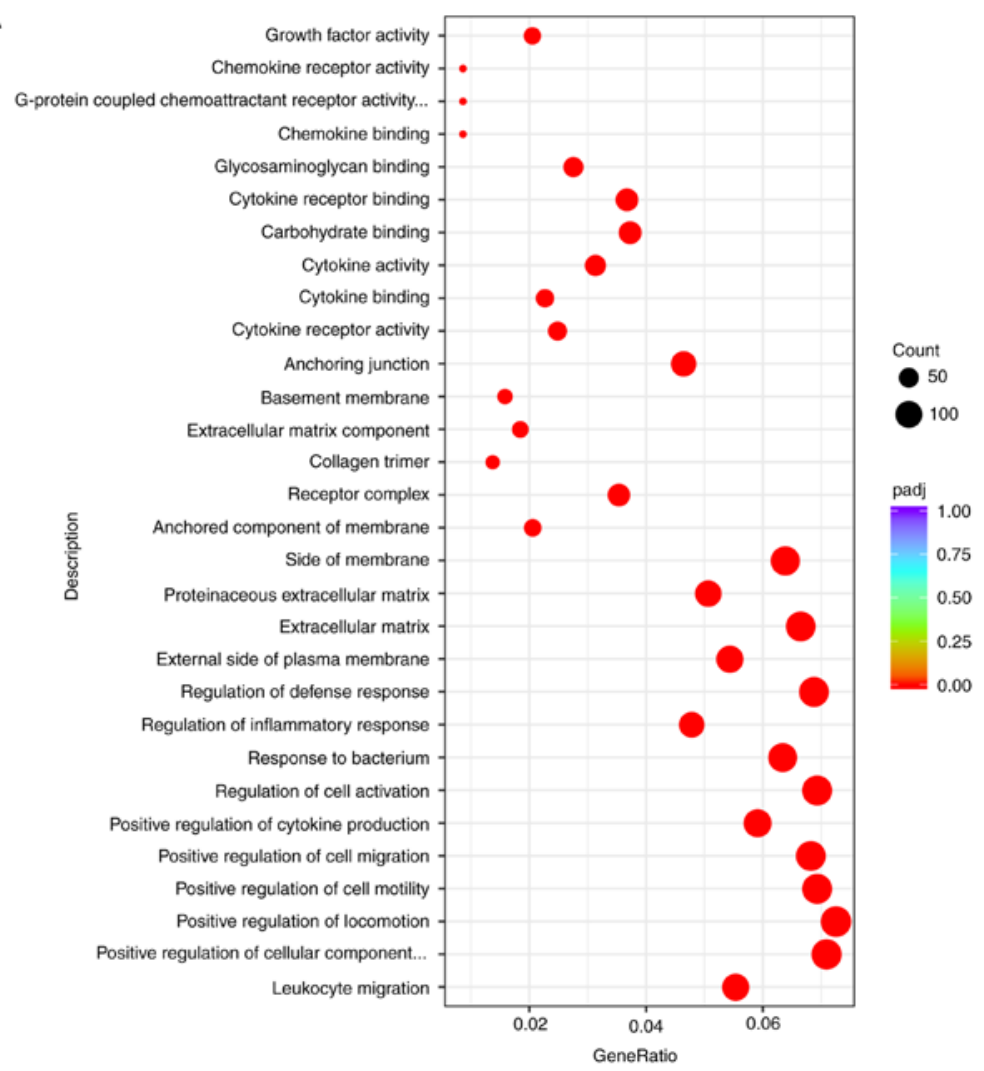

B

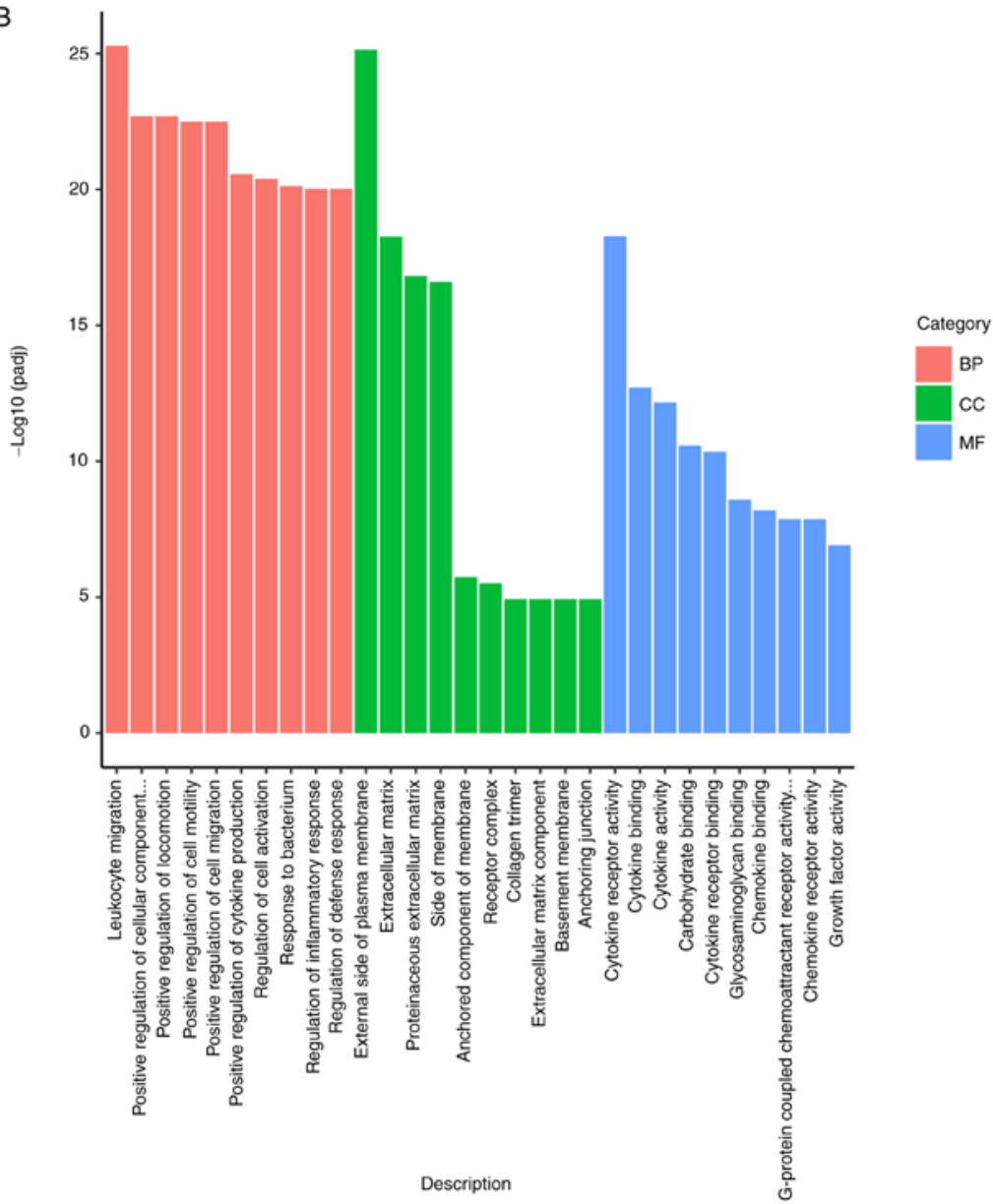

Figure 4. Bubble diagram and bar diagram of the DEG GO terms. Bubble diagram of the top 20 ranked GO terms of the DEGs. In the bubble diagram, the vertical axis indicates GO terms and the horizontal axis represents the enrichment factor. The sizes of dots indicate the number of genes in the GO term. In the bar diagram, GO terms were divided into three categories: The red bar represents BP, the green bar represents CC and the blue bar indicates MF. (A) Bubble diagram of the top 30 ranked DEGs from the comparison between the Blank group and the DVT group. (B) Bar diagram of GO terms from the comparison between the Blank group and the DVT group. 

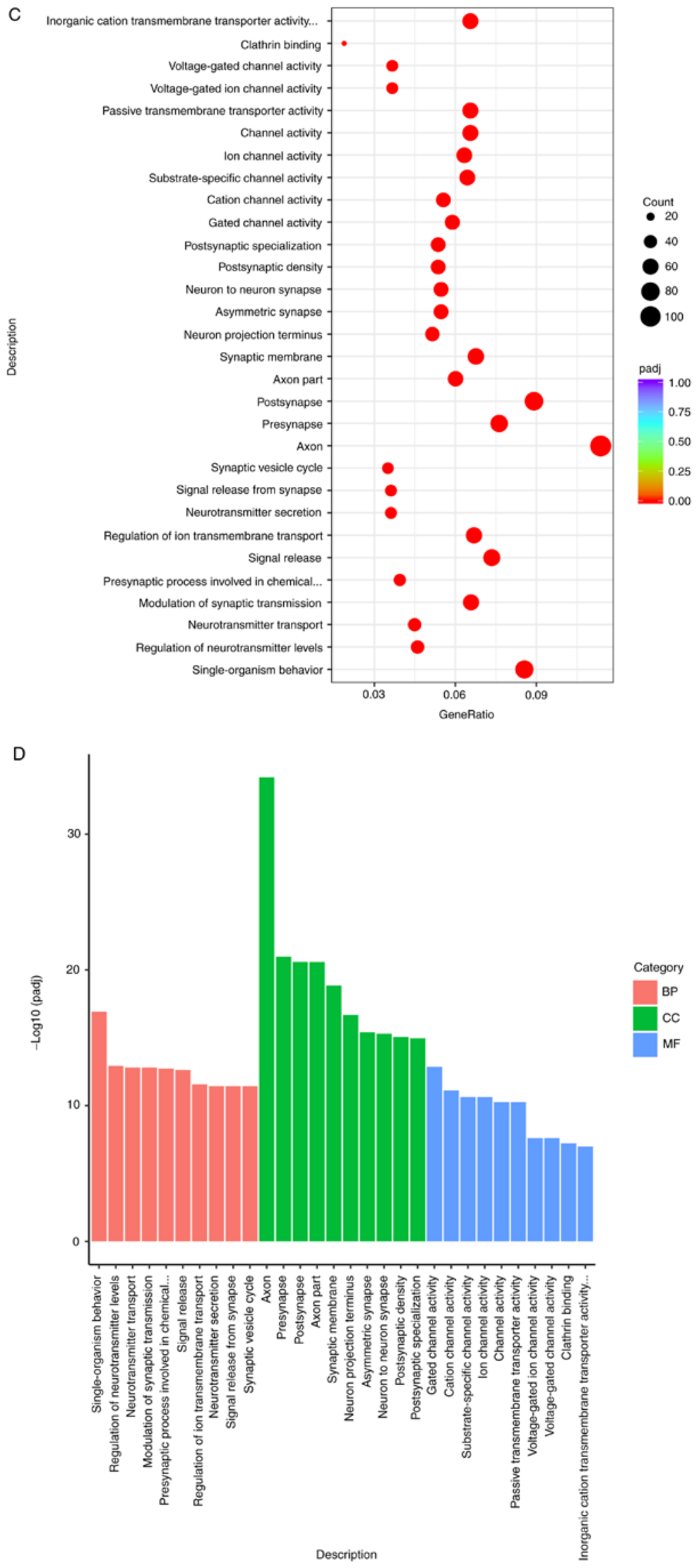

Figure 4. Continued. Bubble diagram and bar diagram of the DEG GO terms. Bubble diagram of the top 20 ranked GO terms of the DEGs. In the bubble diagram, the vertical axis indicates GO terms and the horizontal axis represents the enrichment factor. The sizes of dots indicate the number of genes in the GO term. In the bar diagram, GO terms were divided into three categories: The red bar represents BP, the green bar represents CC and the blue bar indicates MF. (C) Bubble diagram of the top 30 ranked DEGs from the comparison of the Klf15-NC group and the Klf15-siRNA group. (D) Bar diagram of GO terms from the comparison of the Klf15-NC group and the Klf15-siRNA group. si, small interfering; Klf15, Krüppel-like family 15; DVT, deep venous thrombosis; GO, gene ontology; BP, Biological process; MF, molecular function; CC, cellular component; DEGs, differentially expressed genes. 

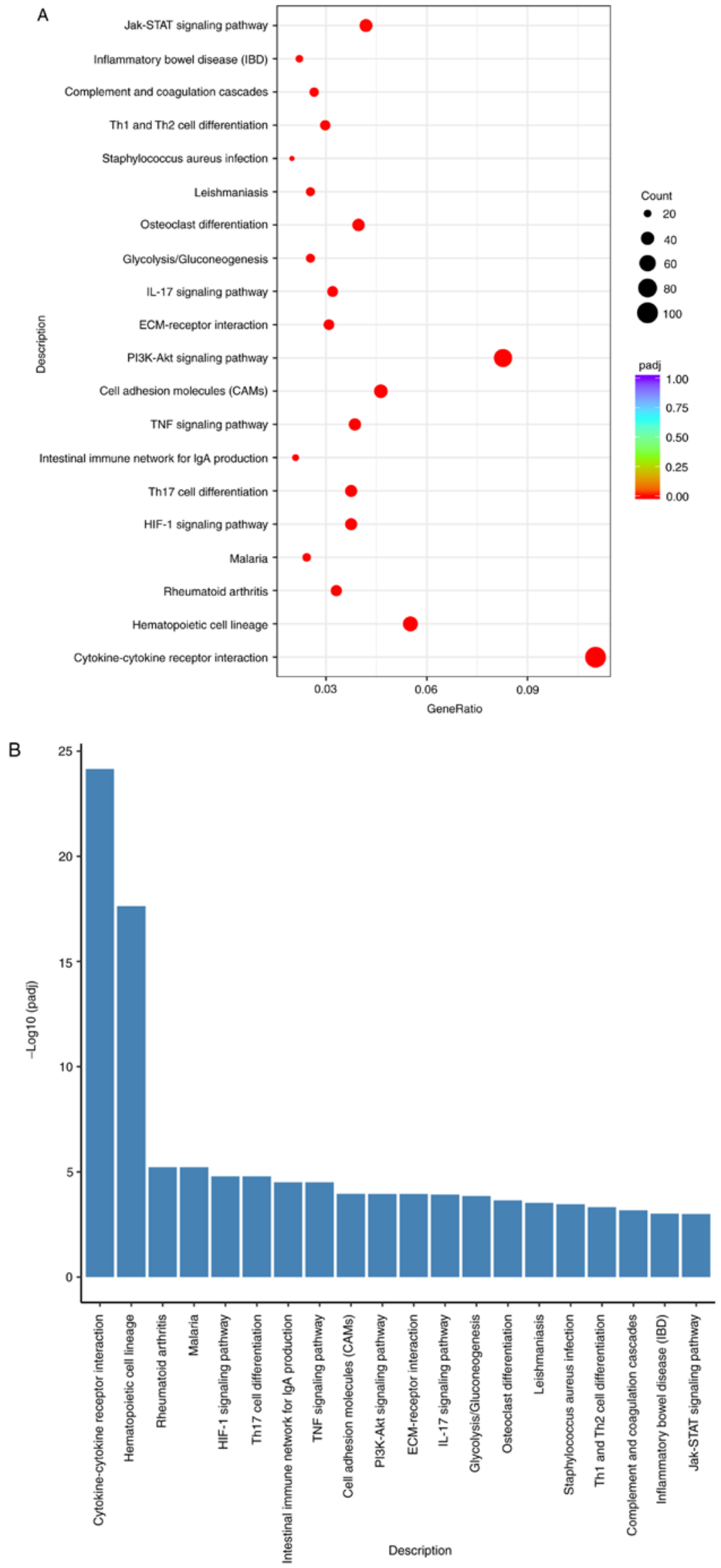

Figure 5. Bar diagram and bubble diagram of KEGG pathway enrichment analysis of DEGs. In the bar diagram, KEGG pathways are listed in the order of the enrichment ratio. A bubble diagram of the top 20 ranked KEGG pathways of DEGs. In the bubble diagram, the vertical axis indicates the KEGG pathways and the horizontal axis represents the enrichment ratio. The sizes of the dots indicate the number of genes in the Gene Ontology term. (A) Bar diagram of KEGG pathways from the comparison between the Blank group and the DVT group. (B) Bubble diagram of the top 20 ranked KEGG pathways from the comparison between the Blank group and the DVT group. 

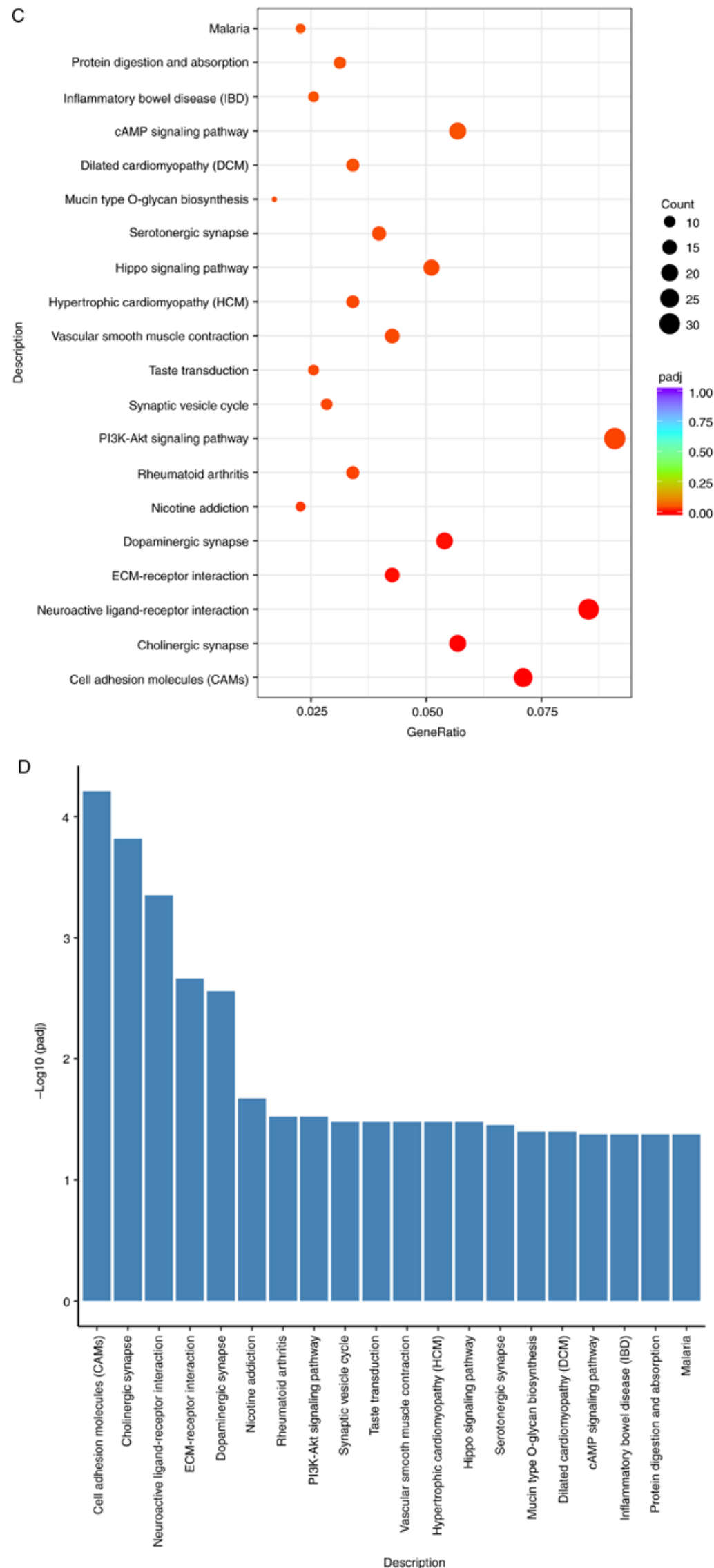

Figure 5. Continued. Bar diagram and bubble diagram of KEGG pathway enrichment analysis of DEGs. In the bar diagram, KEGG pathways are listed in the order of the enrichment ratio. A bubble diagram of the top 20 ranked KEGG pathways of DEGs. In the bubble diagram, the vertical axis indicates the KEGG pathways and the horizontal axis represents the enrichment ratio. The sizes of the dots indicate the number of genes in the Gene Ontology term. (C) Bar diagram of KEGG pathways from the comparison between the Klf15-NC group and the Klf15-siRNA group. (D) Bubble diagram of the top 20 ranked KEGG pathways from the comparison between the Klf15-NC group and the Klf15-siRNA group. si, small interfering; Klf15, Krüppel-like family 15; DVT, deep venous thrombosis; KEGG, Kyoto Encyclopedia of Genes and Genomes. 
A

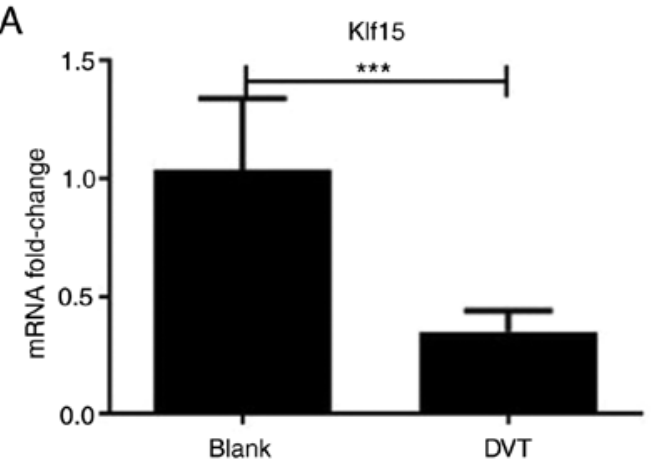

C

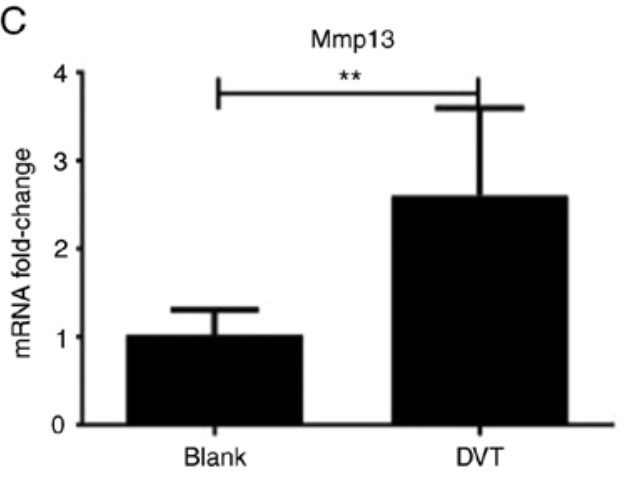

E

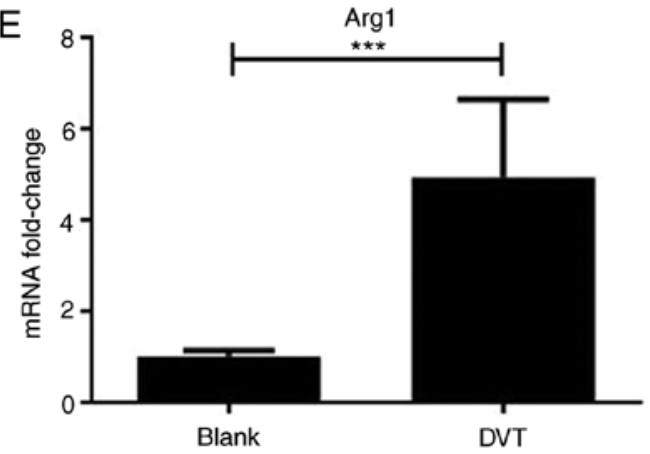

G

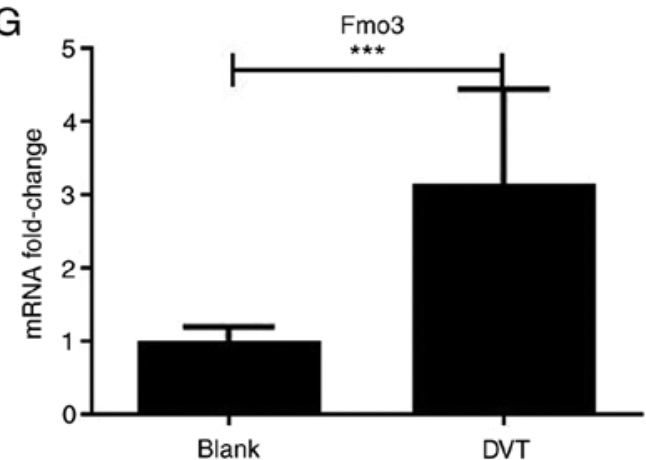

B

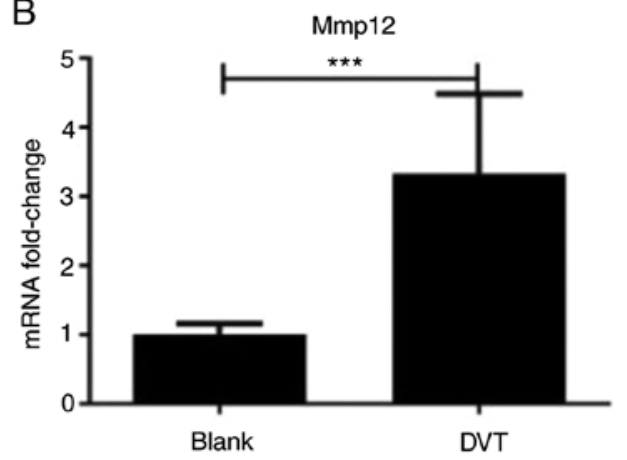

D

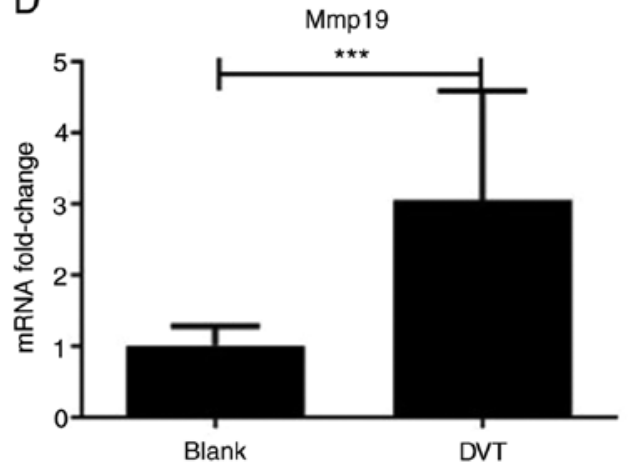

$\mathrm{F}$
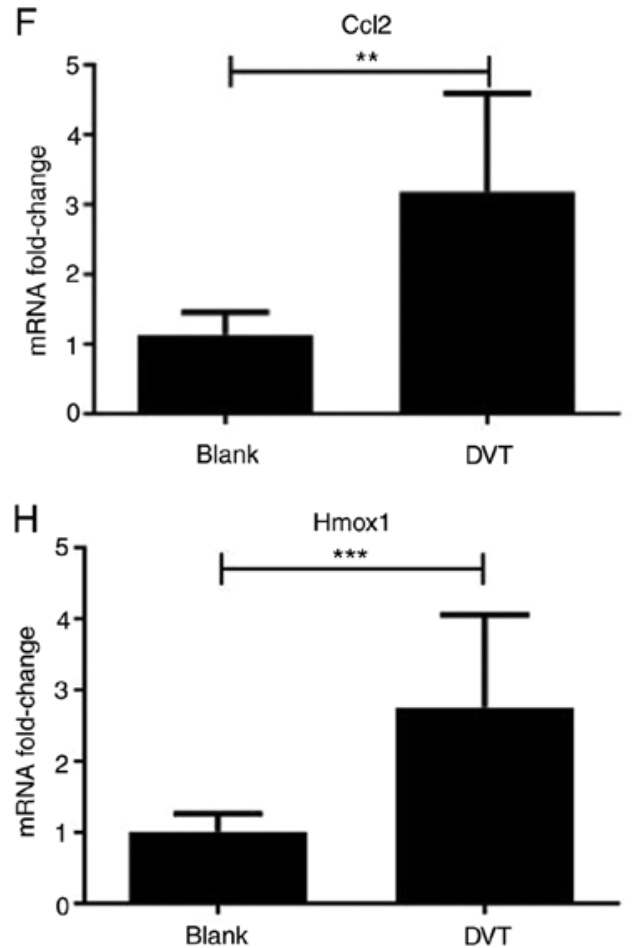

Figure 6. Quantitative PCR analysis of the expression of 7 differentially expressed genes ${ }^{* *} \mathrm{P}<0.01$ and ${ }^{* * * *} \mathrm{P}<0.001$. The expression trends of the detected genes were consistent with those obtained by RNA-sequencing, including the upregulated genes (Mmp13, Mmp12, Mmp19, Arg1, Hmox1 and Fmo3) and downregulated gene (Klf15). (A) Compared with that in Blank group, the expression level of Klf15 decreased significantly in DVT group. ${ }^{* * *} \mathrm{P}<0.001$. (B) The expression level of Mmp12 increased significantly in DVT group than that in Blank group. ${ }^{* * *} \mathrm{P}<0.001$. (C) The expression level of Mmp13 increased significantly in DVT group than that in Blank group. ${ }^{* *} \mathrm{P}<0.01$. (D) The expression level of Mmp19 increased significantly in DVT group than that in Blank group. ${ }^{* * * *} \mathrm{P}<0.001$. (E) Compared with that in Blank group, the expression level of Arg1 increased significantly in DVT group. ${ }^{* * *} \mathrm{P}<0.001$. (F) Compared with that in Blank group, the expression level of Ccl2 increased significantly in DVT group. ${ }^{* *} \mathrm{P}<0.01$. (G) Compared with that in Blank group, the expression level of Fmo3 increased significantly in DVT group. ${ }^{* * *} \mathrm{P}<0.001$. (H) Compared with that in Blank group, the expression level of Hmox 1 increased significantly in DVT group. ${ }^{* * * *} \mathrm{P}<0.001$. Klf15, Krüppel-like family 15; DVT, deep venous thrombosis.

were significantly enriched, including 'the PI3K-Akt signaling pathway', 'the Hippo signaling pathway', 'the cAMP signaling pathway' and 'the relaxin signaling pathway'. In addition, BP terms were identified; these terms included 'the cholinergic synapse', 'neuroactive ligand-receptor interaction', 'ECM-receptor interaction', 'dopaminergic synapse', 
Table I. Number of successful injection of mice and successful modeling of DVT mice.

\begin{tabular}{lcccc}
\hline Group & Blank & DVT & Klf15-NC & Klf15-siRNA \\
\hline Sample size & 10 & 10 & 10 & 10 \\
Number of successful injection of mice & - & - & 9 & 8 \\
Number of successful modeling of DVT mice & - & 7 & 5 & 5 \\
\hline
\end{tabular}

si, small interfering; Klf15, Krüppel-like family 15; DVT, deep venous thrombosis.

Table II. Quality assessment of the raw RNA-sequences reads results of the sequences.

\begin{tabular}{|c|c|c|c|c|c|c|c|}
\hline Sample & Raw_reads & Clean_reads & Clean_bases & Error_rate & Q20 & Q30 & GC_pct \\
\hline DVT1 & 26177314 & 25683459 & $7.71 \mathrm{G}$ & 0.03 & 97.39 & 92.99 & 50.06 \\
\hline DVT2 & 23970627 & 22164628 & $6.65 \mathrm{G}$ & 0.03 & 97.01 & 92.28 & 50.38 \\
\hline DVT3 & 27492800 & 26662217 & $8.0 \mathrm{G}$ & 0.03 & 97.55 & 93.50 & 50.96 \\
\hline Blank1 & 30056705 & 27671970 & $8.3 \mathrm{G}$ & 0.03 & 97.73 & 93.81 & 50.95 \\
\hline Blank2 & 26587894 & 26131760 & $7.84 \mathrm{G}$ & 0.03 & 97.30 & 92.87 & 50.33 \\
\hline Blank3 & 27518969 & 26623013 & $7.99 \mathrm{G}$ & 0.03 & 96.92 & 92.03 & 50.23 \\
\hline Klf15-NC1 & 23873304 & 23344387 & $7.0 \mathrm{G}$ & 0.02 & 98.19 & 94.79 & 50.64 \\
\hline Klf15-NC3 & 31553809 & 31061688 & $9.32 \mathrm{G}$ & 0.02 & 98.28 & 95.07 & 51.05 \\
\hline Klf15-NC4 & 33418796 & 32815907 & $9.84 \mathrm{G}$ & 0.02 & 98.19 & 94.84 & 51.31 \\
\hline Klf15-siRNA_2 & 28443883 & 27658994 & $8.3 \mathrm{G}$ & 0.02 & 98.18 & 94.81 & 50.36 \\
\hline Klf15-siRNA_3 & 31256846 & 30573498 & $9.17 \mathrm{G}$ & 0.02 & 98.26 & 94.90 & 51.13 \\
\hline Klf15-siRNA_5 & 31209875 & 30496859 & $9.15 \mathrm{G}$ & 0.02 & 98.05 & 94.40 & 50.41 \\
\hline
\end{tabular}

si, small interfering; Klf15, Krüppel-like family 15; DVT, deep venous thrombosis; NC, negative control.

Table III. Read mapping results of the sequences

\begin{tabular}{lccccc}
\hline Sample & Total_reads & Total_map (\%) & Unique_map (\%) & Multi_map (\%) & Splice_map (\%) \\
\hline DVT1 & 51366918 & $49046085(95.48)$ & $46528972(90.58)$ & $2517113(4.9)$ & $17368593(33.81)$ \\
DVT2 & 44329256 & $41980259(94.7)$ & $39085822(88.17)$ & $2894437(6.53)$ & $15925579(35.93)$ \\
DVT3 & 53324434 & $50898142(95.45)$ & $47701078(89.45)$ & $3197064(6.0)$ & $19203249(36.01)$ \\
Blank1 & 55343940 & $53021663(95.8)$ & $49481609(89.41)$ & $3540054(6.4)$ & $20032572(36.2)$ \\
Blank2 & 52263520 & $49811834(95.31)$ & $47560000(91.0)$ & $2251834(4.31)$ & $18114051(34.66)$ \\
Blank3 & 53246026 & $50413825(94.68)$ & $48052390(90.25)$ & $2361435(4.43)$ & $18879029(35.46)$ \\
Klf15-NC1 & 46688774 & $45469186(97.39)$ & $42152166(90.28)$ & $3317020(7.1)$ & $16922773(36.25)$ \\
Klf15-NC3 & 62123376 & $60297028(97.06)$ & $55302462(89.02)$ & $4994566(8.04)$ & $21761027(35.03)$ \\
Klf15-NC4 & 65631814 & $63630957(96.95)$ & $58085965(88.5)$ & $5544992(8.45)$ & $23699020(36.11)$ \\
Klf15-siRNA_2 & 55317988 & $53743158(97.15)$ & $50929624(92.07)$ & $2813534(5.09)$ & $19622245(35.47)$ \\
Klf15-siRNA_3 & 61146996 & $59683848(97.61)$ & $53836311(88.04)$ & $5847537(9.56)$ & $22426460(36.68)$ \\
Klf15-siRNA_5 & 60993718 & $59329105(97.27)$ & $55020590(90.21)$ & $4308515(7.06)$ & $21394177(35.08)$ \\
\hline
\end{tabular}

si, small interfering; Klf15, Krüppel-like family 15; NC, negative control; DEG, differentially expressed genes; DVT, deep venous thrombosis.

'nicotine addiction', 'rheumatoid arthritis', 'synaptic vesicle cycle', 'taste transduction', 'vascular smooth muscle contraction', 'hypertrophic cardiomyopathy (HCM)', 'serotonergic synapse', 'mucin type O-glycan biosynthesis', 'dilated cardiomyopathy (DCM)' and 'protein digestion and absorption' terms. Among these terms, the 'vascular smooth muscle contraction', 'HCM' and 'DCM' terms provide crucial information regarding the roles of Klf15 in the formation and pathophysiological processes of vascular disease, especially DVT. 
Table IV. The number of DEGs identified from four groups.

\begin{tabular}{lccc}
\hline DEG set & DEGs & Upregulated & Downregulated \\
\hline DVT_vs_Blank & 2,206 & 1,330 & 876 \\
Klf15-siRNA_vs_NC & 1,041 & 235 & 806 \\
\hline
\end{tabular}

si, small interfering; Klf15, Krüppel-like family 15; DVT, deep venous thrombosis; NC, negative control; DEG, differentially expressed genes.

Table V. DEGs identified in a comparison of the DVT group and the Blank group by RNA-sequencing.

\begin{tabular}{|c|c|c|c|c|c|}
\hline Gene_id & DVT & Blank & $\log 2$ Fold change & Padj & Gene_name \\
\hline ENSMUSG00000049723 & 78.077668 & 47.9396 & 0.707139 & 0.566752 & Mmp12 \\
\hline ENSMUSG00000050578 & 204.5046 & 4.875547 & 5.391707 & $3.03 \times 10^{-09}$ & Mmp13 \\
\hline ENSMUSG00000043613 & 3941.1668 & 290.8774 & 3.759948 & $3.10 \times 10^{-39}$ & Mmp3 \\
\hline ENSMUSG00000005800 & 3114.6048 & 63.94969 & 5.606005 & $1.10 \times 10^{-10}$ & Mmp8 \\
\hline ENSMUSG00000017737 & 7694.2132 & 732.7235 & 3.392543 & $1.06 \times 10^{-29}$ & Mmp9 \\
\hline ENSMUSG00000025355 & 1703.9523 & 203.5787 & 3.064868 & $1.52 \times 10^{-13}$ & Mmp19 \\
\hline ENSMUSG00000026073 & 3607.8063 & 48.75941 & 6.20899 & $3.12 \times 10^{-63}$ & Il1r2 \\
\hline ENSMUSG00000027399 & 553.44397 & 39.4537 & 3.811049 & $9.93 \times 10^{-19}$ & Il1a \\
\hline ENSMUSG00000027398 & 5679.7852 & 139.7907 & 5.344781 & $9.60 \times 10^{-76}$ & Il1b \\
\hline ENSMUSG00000025746 & 1369.395 & 5.758426 & 7.893124 & $2.59 \times 10^{-39}$ & I16 \\
\hline ENSMUSG00000035352 & 906.99266 & 100.0886 & 3.17938 & $6.97 \times 10^{-21}$ & $\mathrm{Ccl12}$ \\
\hline ENSMUSG00000035385 & 3020.6374 & 95.41017 & 4.984662 & $1.14 \times 10^{-08}$ & $\mathrm{Ccl} 2$ \\
\hline ENSMUSG00000094686 & 7.4708094 & 119.9455 & -4.02534 & $2.10 \times 10^{-06}$ & $\mathrm{Ccl} 21 \mathrm{a}$ \\
\hline ENSMUSG00000000982 & 3120.6479 & 31.87038 & 6.61243 & $3.97 \times 10^{-76}$ & $\mathrm{Ccl3}$ \\
\hline ENSMUSG00000018930 & 1205.0351 & 32.31576 & 5.220409 & $1.03 \times 10^{-32}$ & $\mathrm{Ccl} 4$ \\
\hline ENSMUSG00000035042 & 712.57684 & 2817.25 & -1.98337 & $1.21 \times 10^{-11}$ & $\mathrm{Ccl} 5$ \\
\hline ENSMUSG00000018927 & 8371.4662 & 1210.417 & 2.789986 & $1.64 \times 10^{-44}$ & Ccl6 \\
\hline ENSMUSG00000035373 & 2485.7371 & 79.30941 & 4.970069 & $3.32 \times 10^{-07}$ & $\mathrm{Ccl} 7$ \\
\hline ENSMUSG00000019122 & 5063.0309 & 592.4913 & 3.095273 & $2.35 \times 10^{-50}$ & $\mathrm{Ccl} 9$ \\
\hline ENSMUSG00000029380 & 2017.182 & 19.95151 & 6.659775 & $1.9 \times 10^{-104}$ & Cxcl1 \\
\hline ENSMUSG00000061353 & 6200.4582 & 10594.49 & -0.77288 & $4.82 \times 10^{-05}$ & $\mathrm{Cxcl} 12$ \\
\hline ENSMUSG00000021508 & 3590.1969 & 118.9011 & 4.916188 & $6.83 \times 10^{-07}$ & $\mathrm{Cxcl} 14$ \\
\hline ENSMUSG00000018920 & 523.29727 & 975.7794 & -0.89848 & 0.00284 & Cxcl16 \\
\hline ENSMUSG00000058427 & 16451.23 & 10.20436 & 10.65536 & $8.05 \times 10^{-148}$ & $\mathrm{Cxcl} 2$ \\
\hline ENSMUSG00000029379 & 9750.5765 & 0.886821 & 13.4245 & $9.92 \times 10^{-35}$ & $\mathrm{Cxcl} 3$ \\
\hline ENSMUSG00000029371 & 3329.7922 & 4.430165 & 9.552655 & $7.59 \times 10^{-79}$ & $\mathrm{Cxcl5}$ \\
\hline ENSMUSG00000029417 & 721.6049 & 682.6357 & 0.079769 & 0.889672 & Cxcl9 \\
\hline ENSMUSG00000026180 & 3454.5181 & 333.2202 & 3.3741 & $5.89 \times 10^{-46}$ & Cxcr2 \\
\hline ENSMUSG00000045382 & 3109.524 & 812.4151 & 1.93664 & $2.84 \times 10^{-15}$ & Cxcr4 \\
\hline ENSMUSG00000048521 & 152.50759 & 568.3105 & -1.89793 & $1.83 \times 10^{-07}$ & Cxcr6 \\
\hline ENSMUSG00000026691 & 114.11276 & 14.20097 & 3.004407 & 0.005941 & Fmo3 \\
\hline ENSMUSG00000026580 & 1733.686 & 129.0424 & 3.747548 & $5.07 \times 10^{-28}$ & Selp \\
\hline ENSMUSG00000046223 & 3734.2897 & 435.4963 & 3.100282 & $1.12 \times 10^{-47}$ & Plaur \\
\hline ENSMUSG00000005413 & 12311.935 & 735.4273 & 4.065414 & $7.15 \times 10^{-42}$ & Hmox 1 \\
\hline ENSMUSG00000019987 & 9944.886 & 38.99255 & 7.994212 & $1.56 \times 10^{-176}$ & Arg1 \\
\hline
\end{tabular}

si, small interfering; Klf15, Krüppel-like family 15; DVT, deep venous thrombosis.

$q P C R$ validation of DEGs. Next, to confirm the results of the DEG analyses, eight significant DEGs were selected from the RNA-seq results from the comparison of the DVT and Blank groups for further qPCR validation. As shown in Fig. 6, the expression levels of Mmp12 and Mmp13 in the DVT group were significantly increased compared with those in the Blank group. The expression level of K1f15 in the DVT group decreased significantly compared with that in the Blank group. Moreover, 
Table VI. Differentially expressed genes identified in a comparison of the Klf15-siRNA group and the Klf15-NC group.

\begin{tabular}{llcccc}
\hline Gene_id & siRNA & NC & log2 Fold change & Padj & Gene_name \\
\hline ENSMUSG00000030087 & 100.70496 & 301.1934 & -1.58155 & 0.000497 & Klf15 \\
ENSMUSG00000049723 & 647.4817 & 101.9324 & 2.665213 & $6.61 \times 10^{-05}$ & Mmp12 \\
ENSMUSG00000050578 & 401.39705 & 115.9458 & 1.796271 & 0.000549 & Mmp13 \\
\hline
\end{tabular}

si, small interfering; Klf15, Krüppel-like family 15; NC, negative control.

the levels of Mmp 19, Arg1, Ccl2, Fmo3 and Hmox1 in the DVT group were all significantly increased compared with those in the Blank group, which demonstrated the correlation of the results.

\section{Discussion}

DVT is one of the most common vascular diseases and has a high mortality rate (1). Nevertheless, the current diagnostic and therapeutic methods are limited (18). In current DVT studies, the mechanism and regulatory factors involved in the formation and pathological process of DVT should be investigated to provide a foundation for the diagnosis and treatment of DVT (19).

Klf15 was shown to be closely related to cardiovascular diseases such as hypertension, atherosclerosis and coronary heart disease (20). Klf15 is a transcriptional regulatory factor involved in various functions, including cell differentiation, apoptosis and tumor formation, and is expressed in various tissues and organs, including the heart, liver, and kidneys (21). Moreover, Klf15 plays a key role in the development of atherosclerosis (12). According to the authors' preliminary experiments, it was found that Klf15 might also affect thrombosis. To promote knowledge about the relationship and genetic interaction between DVT and Klf15, this study was performed.

Numerous obstacles prevent the complete understanding of the pathology, diagnosis and treatment of DVT. The present study aimed to examine factors regulating the initiation and progression of DVT and factors related to effective and utilizable measures.

To the best of our knowledge, this is the first study to perform high-throughput sequencing in a mouse DVT model and to investigate the effect of Klf15 on DVT formation. The data and analyses in the current study suggest that pathways including TNF, PI3K-Akt, IL-17, Jak-STAT, NF- $\kappa$ B, and MAPK should be considered, as such pathways were correlated with the formation of thrombi according to the KEGG enrichment analysis of the DEGs between the DVT and Blank groups. Previous studies $(22,23)$ have reported that MAPK pathways are related to vascular endothelial venous thrombosis and our colleagues have suggested that resveratrol may exert an in vitro antithrombotic activity by inactivating MAPK signaling pathways (24). Moreover, KEGG analysis of the comparison of the Klf15-siRNA group and the Klf15-NC group indicated that PI3K-Akt play a central role in the regulatory pathway involved in DVT formation.

The DEGs revealed by these comparisons indicated the crucial role of certain genes in the regulation of DVT. In the comparison of the DVT and Blank groups, the identified genes ranged from members of the Mmp family, the IL family, and the chemokine family to Selp, Plaur, Hmox 1 and Arg1. Fonseca et al (25) indicated that Mmp plays a critical role in numerous cellular processes. Li et al (26) discovered that Mmp3 polymorphisms and upregulated protein expression in the Chinese Han population may provide new markers associated with the evaluation of DVT diagnosis and risk. Lenglet et al (27) performed a study on mice by subjecting their brains to ischemic stroke and revealed differentially expressed levels of Mmp family genes, including significantly upregulated expression of Mmp9, 10, and 13 and Timp1. Xiao et al (28) indicated that Mmp8 enhanced vascular smooth muscle cell (VSMC) proliferation and played an important role in neointima formation via ADAM10-, N-cadherin-, and $\beta$-catenin-mediated pathways. Mmp8 enhances VSMC proliferation, according to a study of WT and Mmp9-/- mice that underwent stasis venous thrombosis (VT) by ligation of the IVC. The tissues were harvested at different time points and the results showed that the midterm vein wall collagen content was regulated by Mmp9 (28). Thus, Mmp9 plays a role in both vein wall responses and late thrombus resolution. Quillard et al (29) found that Mmp13 prevailed over Mmp8 in collagen degradation in atheromata, thus identifying a selective target for plaque structure formation. Based on the current analysis and previous reports, the present study believes that the role of the MMP family in DVT deserves further study.

Genes in the IL family were identified, including Il-Ir2, Il-la, Il-lb and Il-6, that showed higher expression in the DVT group than in the Blank group. Gupta et al (30) demonstrated the increased expression of NLRP3, caspase-1 and IL-1 $\beta$ in individuals with clinically established VT. van Minkelen et al (31) found that $I L I R N-\mathrm{H} 5 \mathrm{H} 5$ carriership increases the risk of VT. Analyses of the DEGs in the chemokine families revealed that these DEGs had generally higher expression in the DVT group than in the Blank group, with the exception of some members mentioned in qPCR Validation of DEGs. Among those genes in the $\mathrm{Cc}$ and $\mathrm{Cx}$ families, a study of Cxcr2 was previously performed. Henke et al (32) found that normal DVT resolution involved Cxcr2-mediated neovascularization, collagen turnover and fibrinolysis and that this process is probably primarily monocyte-dependent. Henke and Wakefield (33) indicated that early thrombus resolution primarily involves Cxcr2-associated plasmin activation and Mmp-9, while later resolution involves both Cxcr2- and Ccr2-mediated uPA cell influx and thrombus angiogenesis. According to the above reports and our data, inflammation plays an important role in the formation of DVT. The present study speculated that $\mathrm{Ccl} 2$, a downstream gene of Klf15, may be the key factor in the effects of Klf15 on DVT formation. 
Table VII. KEGG pathway enrichment analysis of the DVT group vs. the Blank group.

\begin{tabular}{|c|c|c|c|c|c|c|c|}
\hline KEGGID & Description & Gene ratio & BgRatio & P-value & Count & Up & Down \\
\hline mmu04060 & Cytokine-cytokine receptor interaction & $100 / 907$ & $234 / 6352$ & $2.48 \times 10^{-27}$ & 100 & 60 & 40 \\
\hline mmu04640 & Hematopoietic cell lineage & $50 / 907$ & $89 / 6352$ & $1.65 \times 10^{-20}$ & 50 & 26 & 24 \\
\hline mmu05323 & Rheumatoid arthritis & $30 / 907$ & $77 / 6352$ & $7.01 \times 10^{-08}$ & 30 & 21 & 9 \\
\hline mmu05144 & Malaria & $22 / 907$ & $47 / 6352$ & $8.39 x^{-08}$ & 22 & 20 & 2 \\
\hline mmu04066 & HIF-1 signaling pathway & $34 / 907$ & $99 / 6352$ & $3.48 \times 10^{-07}$ & 34 & 31 & 3 \\
\hline mmu04659 & Th17 cell differentiation & $34 / 907$ & $99 / 6352$ & $3.48 \times 10^{-07}$ & 34 & 13 & 21 \\
\hline mmu04672 & Intestinal immune network for $\operatorname{Ig} \mathrm{A}$ production & $19 / 907$ & $41 / 6352$ & $7.86 \times 10^{-07}$ & 19 & 4 & 15 \\
\hline mmu04668 & TNF signaling pathway & $35 / 907$ & $107 / 6352$ & $8.86 \times 10^{-07}$ & 35 & 32 & 3 \\
\hline mmu04514 & Cell adhesion molecules & $42 / 907$ & $146 / 6352$ & $3.46 \times 10^{-06}$ & 42 & 18 & 24 \\
\hline mmu04151 & PI3K-Akt signaling pathway & $75 / 907$ & $319 / 6352$ & $4.15 \times 10^{-06}$ & 75 & 51 & 24 \\
\hline mmu04512 & ECM-receptor interaction & $28 / 907$ & $82 / 6352$ & $4.28 \times 10^{-06}$ & 28 & 19 & 9 \\
\hline mmu04657 & IL-17 signaling pathway & $29 / 907$ & $87 / 6352$ & $5.01 \times 10^{-06}$ & 29 & 27 & 2 \\
\hline mmu00010 & Glycolysis/gluconeogenesis & $23 / 907$ & $62 / 6352$ & $6.36 \times 10^{-06}$ & 23 & 19 & 4 \\
\hline mmu04380 & Osteoclast differentiation & $36 / 907$ & $123 / 6352$ & $1.13 \times 10^{-05}$ & 36 & 34 & 2 \\
\hline mmu05140 & Leishmaniasis & $23 / 907$ & $65 / 6352$ & $1.58 \times 10^{-05}$ & 23 & 17 & 6 \\
\hline mmu05150 & Staphylococcus aureus infection & $18 / 907$ & $45 / 6352$ & $1.95 \times 10^{-05}$ & 18 & 12 & 6 \\
\hline mmu04658 & Th1 and Th2 cell differentiation & $27 / 907$ & $85 / 6352$ & $2.85 \times 10^{-05}$ & 27 & 7 & 20 \\
\hline mmu04610 & Complement and coagulation cascades & $24 / 907$ & $73 / 6352$ & $4.20 \times 10^{-05}$ & 24 & 19 & 5 \\
\hline mmu05321 & Inflammatory bowel disease & 20/907 & $57 / 6352$ & $6.44 \times 10^{-05}$ & 20 & 10 & 10 \\
\hline mmu04630 & Jak-STAT signaling pathway & $38 / 907$ & $143 / 6352$ & $7.05 \times 10^{-05}$ & 38 & 25 & 13 \\
\hline mmu05152 & Tuberculosis & $41 / 907$ & $160 / 6352$ & $9.04 \times 10^{-05}$ & 41 & 29 & 12 \\
\hline mmu04064 & $\mathrm{NF}-\kappa \mathrm{B}$ signaling pathway & $27 / 907$ & $92 / 6352$ & 0.0001319 & 27 & 16 & 11 \\
\hline mmu05340 & Primary immunodeficiency & $14 / 907$ & $35 / 6352$ & 0.0001645 & 14 & 0 & 14 \\
\hline mmu05202 & Transcriptional misregulation in cancer & $41 / 907$ & $169 / 6352$ & 0.0003243 & 41 & 27 & 14 \\
\hline mmu05162 & Measles & $32 / 907$ & $122 / 6352$ & 0.0003335 & 32 & 19 & 13 \\
\hline mmu04010 & MAPK signaling pathway & $61 / 907$ & $281 / 6352$ & 0.0003692 & 61 & 49 & 12 \\
\hline mmu05166 & HTLV-I infection & $57 / 907$ & $262 / 6352$ & 0.0005396 & 57 & 31 & 26 \\
\hline mmu05164 & Influenza A & $36 / 907$ & $147 / 6352$ & 0.0006105 & 36 & 27 & 9 \\
\hline mmu00052 & Galactose metabolism & $12 / 907$ & $31 / 6352$ & 0.0006857 & 12 & 11 & 1 \\
\hline mmu05418 & Fluid shear stress and atherosclerosis & $34 / 907$ & $138 / 6352$ & 0.0007639 & 34 & 31 & 3 \\
\hline mmu05320 & Autoimmune thyroid disease & $16 / 907$ & $50 / 6352$ & 0.0010955 & 16 & 6 & 10 \\
\hline mmu00220 & Arginine biosynthesis & $8 / 907$ & $17 / 6352$ & 0.001229 & 8 & 6 & 2 \\
\hline mmu05145 & Toxoplasmosis & $27 / 907$ & $105 / 6352$ & 0.0013127 & 27 & 15 & 12 \\
\hline mmu04062 & Chemokine signaling pathway & $40 / 907$ & $176 / 6352$ & 0.0015037 & 40 & 27 & 13 \\
\hline mmu04216 & Ferroptosis & $13 / 907$ & $39 / 6352$ & 0.0020876 & 13 & 11 & 2 \\
\hline mmu04612 & Antigen processing and presentation & $20 / 907$ & $74 / 6352$ & 0.0028603 & 20 & 9 & 11 \\
\hline mmu05332 & Graft-versus-host disease & $15 / 907$ & $50 / 6352$ & 0.0031691 & 15 & 7 & 8 \\
\hline mmu05133 & Pertussis & $19 / 907$ & $70 / 6352$ & 0.0034227 & 19 & 17 & 2 \\
\hline mmu04145 & Phagosome & $35 / 907$ & $156 / 6352$ & 0.0036189 & 35 & 25 & 10 \\
\hline mmu04621 & NOD-like receptor signaling pathway & $34 / 907$ & $151 / 6352$ & 0.0038603 & 34 & 30 & 4 \\
\hline mmu05134 & Legionellosis & $16 / 907$ & $56 / 6352$ & 0.0040295 & 16 & 15 & 1 \\
\hline mmu00590 & Arachidonic acid metabolism & $17 / 907$ & $61 / 6352$ & 0.0040877 & 17 & 14 & 3 \\
\hline mmu04620 & Toll-like receptor signaling pathway & $22 / 907$ & $87 / 6352$ & 0.0044241 & 22 & 19 & 3 \\
\hline mmu05230 & Central carbon metabolism in cancer & $17 / 907$ & $62 / 6352$ & 0.0049021 & 17 & 16 & 1 \\
\hline mmu05330 & Allograft rejection & $14 / 907$ & $48 / 6352$ & 0.0056832 & 14 & 4 & 10 \\
\hline mmu04940 & Type I diabetes mellitus & $15 / 907$ & $53 / 6352$ & 0.0058193 & 15 & 6 & 9 \\
\hline mmu04931 & Insulin resistance & $25 / 907$ & $105 / 6352$ & 0.0058616 & 25 & 18 & 7 \\
\hline mmu05310 & Asthma & $8 / 907$ & $21 / 6352$ & 0.0060726 & 8 & 1 & 7 \\
\hline mmu05142 & Chagas disease (American trypanosomiasis) & 24/907 & $100 / 6352$ & 0.0061752 & 24 & 18 & 6 \\
\hline mmu01230 & Biosynthesis of amino acids & $18 / 907$ & $70 / 6352$ & 0.0079558 & 18 & 15 & 3 \\
\hline
\end{tabular}


Table VIII. KEGG Pathways enrichment analysis of KLF15-siRNA group vs. KLF15-NC group.

\begin{tabular}{|c|c|c|c|c|c|c|c|}
\hline KEGGID & Description & Gene ratio & BgRatio & P-value & Count & Up & Down \\
\hline mmu04514 & Cell adhesion molecules & $25 / 352$ & $144 / 6351$ & $2.50 \times 10^{-07}$ & 25 & 8 & 17 \\
\hline $\mathrm{mmu} 04725$ & Cholinergic synapse & $20 / 352$ & $107 / 6351$ & $1.23 \times 10^{-06}$ & 20 & 0 & 20 \\
\hline mmu04080 & Neuroactive ligand-receptor interaction & $30 / 352$ & $225 / 6351$ & $5.42 \times 10^{-06}$ & 30 & 3 & 27 \\
\hline mmu04512 & ECM-receptor interaction & $15 / 352$ & $82 / 6351$ & $3.52 \times 10^{-05}$ & 15 & 0 & 15 \\
\hline mmu04728 & Dopaminergic synapse & $19 / 352$ & $126 / 6351$ & $5.60 \times 10^{-05}$ & 19 & 0 & 19 \\
\hline mmu05033 & Nicotine addiction & $8 / 352$ & $35 / 6351$ & 0.0005167 & 8 & 0 & 8 \\
\hline mmu05323 & Rheumatoid arthritis & $12 / 352$ & $76 / 6351$ & 0.0008645 & 12 & 8 & 4 \\
\hline mmu04151 & PI3K-Akt signaling pathway & $32 / 352$ & $325 / 6351$ & 0.0009717 & 32 & 5 & 27 \\
\hline mmu04721 & Synaptic vesicle cycle & $10 / 352$ & $59 / 6351$ & 0.0013226 & 10 & 1 & 9 \\
\hline mmu04742 & Taste transduction & $9 / 352$ & $50 / 6351$ & 0.0014766 & 9 & 0 & 9 \\
\hline mmu04270 & Vascular smooth muscle contraction & $15 / 352$ & $115 / 6351$ & 0.0015912 & 15 & 1 & 14 \\
\hline mmu05410 & Hypertrophic cardiomyopathy & $12 / 352$ & $82 / 6351$ & 0.0017112 & 12 & 1 & 11 \\
\hline mmu04390 & Hippo signaling pathway & $18 / 352$ & $152 / 6351$ & 0.0017475 & 18 & 1 & 17 \\
\hline mmu04726 & Serotonergic synapse & $14 / 352$ & $106 / 6351$ & 0.0020024 & 14 & 0 & 14 \\
\hline mmu00512 & Mucin type O-glycan biosynthesis & $6 / 352$ & $26 / 6351$ & 0.0024822 & 6 & 1 & 5 \\
\hline mmu05414 & Dilated cardiomyopathy & $12 / 352$ & $86 / 6351$ & 0.0025876 & 12 & 0 & 12 \\
\hline mmu04024 & cAMP signaling pathway & $20 / 352$ & $186 / 6351$ & 0.003181 & 20 & 0 & 20 \\
\hline mmu05321 & Inflammatory bowel disease & $9 / 352$ & $56 / 6351$ & 0.003331 & 9 & 7 & 2 \\
\hline mmu04974 & Protein digestion and absorption & $11 / 352$ & $78 / 6351$ & 0.0035469 & 11 & 2 & 9 \\
\hline mmu05144 & Malaria & $8 / 352$ & $47 / 6351$ & 0.0038607 & 8 & 2 & 6 \\
\hline mmu04727 & GABAergic synapse & $11 / 352$ & $79 / 6351$ & 0.0039219 & 11 & 0 & 11 \\
\hline mmu04911 & Insulin secretion & $11 / 352$ & $79 / 6351$ & 0.0039219 & 11 & 1 & 10 \\
\hline mmu04926 & Relaxin signaling pathway & $15 / 352$ & $126 / 6351$ & 0.0039259 & 15 & 1 & 14 \\
\hline
\end{tabular}

KEGG, Kyoto Encyclopedia of Genes and Genomes; DVT, deep venous thrombosis; si, small interfering; NC, negative control.

A study of the relationship between Hmox1 and DVT was conducted by Bean et al (34) who identified a critical cytoprotective enzyme encoded by the inducible Hmox 1 gene with anti-inflammatory, antioxidant and anticoagulant activities in the vascular system. A (GT)n microsatellite located in the promoter of the Hmox1 gene influences the level of the response. Peng et al (35) stimulated HO-1 (Hmox1) production and revealed the inhibition of platelet-dependent thrombus formation in $\mathrm{HO}^{-1-}{ }^{--}$mice compared with that in WT mice, and this inhibition may represent an adaptive response mechanism to reduce platelet activation.

Bojic et al (36) conducted a study on mice regarding the relationships between the peroxisome proliferator-activated receptor (PPAR) $\delta$ agonist GW1516 in aortic inflammation and atherosclerosis via intervention by the PPAR $\delta$ agonist; this study revealed that the progression of preestablished atherosclerosis was inhibited by aortic inflammation and attenuated by the progression of preestablished atherosclerosis. Furthermore, GW1516 intervention decreased the expression of aortic proinflammatory M1 cytokines, increased the expression of the anti-inflammatory M2 cytokine Arg1 and attenuated the iNos/Argl ratio. Samsoondar et al (37) performed hepatic gene expression analysis on $L d l r-/$ - mice fed a high-fat, high-cholesterol diet $(42 \% \mathrm{kcal}$ fat, $0.2 \%$ cholesterol) supplemented with bempedoic acid at $0,3,10$ and $30 \mathrm{mg} / \mathrm{kg}$ body weight. These results showed that in the full-length aorta, bempedoic acid markedly suppressed cholesteryl ester accumulation, attenuated the expression of proinflammatory M1 genes and attenuated the $i \mathrm{Nos} / \mathrm{Arg} 1 \mathrm{ratio}$, which demonstrated that $\mathrm{Ccl} 3$ and Nos2 are marker genes for M1 macrophages and that Arg1 may be a marker gene for M2 macrophages. To the best of our knowledge, research and papers on the role of Arg1 in thrombosis are scarce but based on the current data, it suggests that Arg1 should be studied.

The present study first identified the critical roles of $\mathrm{Fmo3}$ and Mmp19 in regulating DVT. Zhu et al (38) demonstrated that the microbe-dependent production of trimethylamine $\mathrm{N}$-oxide (TMAO) contributes to the risk of thrombosis. Thus, a murine $\mathrm{FeCl}_{3}$-induced carotid artery injury model was established to confirm the impact of FMO3 suppression [via antisense oligonucleotide (ASO) targeting] and overexpression (as a transgene), which was examined by the plasma TMAO levels, platelet responsiveness and thrombosis potential. The present study demonstrated that host hepatic FMO3, the final product of the metaorganismal TMAO pathway, participates in diet- and gut microbiota-dependent changes in both platelet responsiveness and thrombosis potential in vivo (37). Shih et al (39) treated WT and FMO3KO mice with control or FMO-3 ASOs. FMO-3-ASO treatment led to the same extent of lipid-lowering effects in the FMO3KO mice as it did in the WT mice, indicating off-target effects. This study revealed that both FMO3KO and WT mice fed a $0.5 \%$ choline diet showed a substantial reduction in both TMAO and in vivo thrombosis potential. 
In conclusion, a transcriptome study consisting of two parts was performed to investigate the expression levels of Klf15 and other related genes in C57BL/6 mice with IVC thrombi for the first time. The experimental results indicated that 2,206 genes were differentially expressed between the DVT group and the Blank group, and 1,041 DEGs were identified by comparing the Klf15-siRNA group with the Klf15-NC group. The present study confirmed that $\mathrm{Arg} 1, \mathrm{Ccl} 2$ and Hmox1 are related to DVT, as previously identified, and new genes related to the formation of DVT were identified, including Fmo3 and Mmp19. Furthermore, to the best of our knowledge, the present study is the first to reveal that genes such as Mmp12 and Mmp13 are involved in the regulation of DVT; the current results obtained via comparison of the Klf15-siRNA group and the Klf15-NC group are especially revealing. Given the data obtained in the present experiments, it is speculated that Klf15 may play a role in DVT by regulating inflammatory genes, some members of the MMP family or other DEGs; however, this speculation needs to be confirmed in the future. In the next study, cell experiments, clinical experiments and additional animal experiments will be performed to confirm the role of Klf15 in DVT, including pathway regulation and whether DVT formation is regulated by Klf15 via Mmp12 and Mmp13. The present research provides new insights and prospects for studying the mechanism of thrombosis and possible drug targets.

\section{Acknowledgements}

Not applicable.

\section{Funding}

The present study was supported by the National Natural Science Foundation of China (Grant. Nos. 81760029 and 81760030) and the Health Science and Technology Project of Yunnan Province (Grant. Nos. 2017NS021 and 2018NS0106).

\section{Availability of data and materials}

The datasets used and/or analyzed during the current study are available from the corresponding author upon reasonable request.

\section{Authors' contributions}

$\mathrm{XZ}$ and RZ conceived the present study. RZ designed the experiments. JZ and SX prepared the materials and conducted the experiments on mice with IVC thrombi. XZ processed the data. RZ contributed substantially to the data analysis and manuscript revision. All authors read and approved the final manuscript.

\section{Ethics approval and consent to participate}

All animal experiments were performed following approval from the Animal Experimental Ethical Committee of Kunming Medical University (Kunming, China).

\section{Patient consent for publication}

Not applicable.

\section{Competing interest}

The authors declare that they have no conflicts of interest.

\section{References}

1. Huerta C, Johansson S, Wallander MA and García Rodríguez LA: Risk factors and short-term mortality of venous thromboembolism diagnosed in the primary care setting in the United Kingdom. Arch Intern Med 167: 935-943, 2007.

2. Zhu L, Cheng J, Gu P, Liu Y, Liu J, Wang J and Shen H: Therapeutic strategies of thromboembolic events in patients with inflammatory bowel diseases: Two case reports. Medicine (Baltimore) 98: e14622, 2019.

3. Kandic Z, Firdus N, Kandic A, Catic L and Kandic E: Treatment of the rectal cancer in casuistic clinic for abdominal surgery, Clinical centre of the University of Sarajevo (2006-2010.). Acta Chir Iugosl 59: 97-101, 2012.

4. Leenders JJ, Wijnen WJ, van der Made I, Hiller M, Swinnen M, Vandendriessche T, Chuah M, Pinto YM and Creemers EE: Repression of cardiac hypertrophy by KLF15: Underlying mechanisms and therapeutic implications. PLoS One 7: e36754, 2012.

5. Payne H, Ponomaryov T, Watson SP and Brill A: Mice with a deficiency in CLEC-2 are protected against deep vein thrombosis. Blood 129: 2013-2020, 2017

6. Brill A, Fuchs TA, Chauhan AK, Yang JJ, De Meyer SF, Köllnberger M, Wakefield TW, Lämmle B, Massberg S and Wagner DD: Von Willebrand factor-mediated platelet adhesion is critical for deep vein thrombosis in mouse models. Blood 117: 1400-1407, 2011

7. Fan L, Hsieh PN, Sweet DR and Jain MK: Krüppel-like factor 15: Regulator of BCAA metabolism and circadian protein rhythmicity. Pharmacol Res 130: 123-126, 2018.

8. Lu Y, Zhang L, Liao X, Sangwung P, Prosdocimo DA, Zhou G, Votruba AR, Brian L, Han YJ, Gao H, et al: Kruppel-like factor 15 is critical for vascular inflammation. J Clin Invest 123: 4232-4241, 2013.

9. Trapnell C, Pachter L and Salzberg SL: TopHat: Discovering splice junctions with RNA-Seq. Bioinformatics 25: 1105-1111, 2009.

10. Cui JY and Klaassen CD: RNA-Seq reveals common and unique PXR-and CAR-target gene signatures in the mouse liver transcriptome. Biochim Biophys Acta 1859: 1198-1217, 2016.

11. Lu Y, Haldar S, Croce K, Wang Y, Sakuma M, Morooka T, Wang B, Jeyaraj D, Gray SJ, Simon DI and Jain MK: Kruppel-like factor 15 regulates smooth muscle response to vascular injury-brief report. Arterioscler Thromb Vasc Biol 30: 1550-1552, 2010.

12. Liu B, Xu L, Yu X, Li W, Sun X, Xiao S, Guo M and Wang H: Protective effect of KLF15 on vascular endothelial dysfunction induced by TNF- $\alpha$. Mol Med Rep 18: 1987-1994, 2018.

13. Mackman N: Triggers, targets and treatments for thrombosis. Nature 451: 914-918, 2008.

14. Yuping Y, Hua C and Qing Z: Advances in the relationship between Kruppel-like factor 15 and cardiovascular disease research. Cardiovasc Endocrinol Metab 7: 37-41, 2018.

15. Mackman N: New insights into the mechanisms of venous thrombosis. J Clin Invest 122: 2331-2336, 2012.

16. Li G,Zhou R, Zhao X, Liu R and Ye C: [Corrigendum] Correlation between the expression of IL-18 and deep venous thrombosis. Int J Mol Med: August 27, 2018 (Epub ahead of print).

17. Yu G, Wang LG, Han Y and He QY: ClusterProfiler: An R package for comparing biological themes among gene clusters. OMICS 16: 284-287, 2012.

18. Lee AY and Hirsh J: Diagnosis and treatment of venous thromboembolism. Annu Rev Med 53: 15-33, 2002.

19. Machlus KR, Aleman MM and Wolberg AS: Update on venous thromboembolism: Risk factors, mechanisms, and treatments. Arterioscler Thromb Vasc Biol 31: 476-478, 2011.

20. Haldar SM, Lu Y, Jeyaraj D, Kawanami D, Cui Y, Eapen SJ, Hao C, Li Y, Doughman YQ, Watanabe M, et al: Klf15 deficiency is a molecular link between heart failure and aortic aneurysm formation. Sci Transl Med 2: 26ra26, 2010.

21. Noack C, Haupt LP, Zimmermann WH, Streckfuss-Bömeke K and Zelarayán LC: Generation of a KLF15 homozygous knockout human embryonic stem cell line using paired CRISPR/Cas9n, and human cardiomyocytes derivation. Stem Cell Res 23: 127-131, 2017. 
22. Jiang Z, Ma J, Wang Q, Wu F, Ping J and Ming L: Circulating microRNA expression and their target genes in deep vein thrombosis: A systematic review and bioinformatics analysis. Medicine (Baltimore) 96: e9330, 2017.

23. Noble S and Pasi J: Epidemiology and pathophysiology of cancer-associated thrombosis. Br J Cancer 102 (Suppl 1): S2-S9, 2010.

24. Lou Z, Li X, Zhao X, Du K, Li X and Wang B: Resveratrol attenuates hydrogen peroxide-induced apoptosis, reactive oxygen species generation, and PSGL-1 and VWF activation in human umbilical vein endothelial cells, potentially via MAPK signalling pathways. Mol Med Rep 17: 2479-2487, 2018.

25. Fonseca FL, da Costa Aguiar Alves B, Azzalis LA and Belardo TM: Matrix metalloproteases as biomarkers of disease. Methods Mol Biol 1579: 299-311, 2017.

26. Li XP, Wan GZ, Wang GJ and Li JF: MMP3-1171 5A/6A promoter genotype influences serum MMP3 levels and is associated with deep venous thrombosis. Ann Vasc Surg 34: 261-267, 2016.

27. Lenglet S, Montecucco F, Mach F, Schaller K, Gasche Y and Copin JC: Analysis of the expression of nine secreted matrix metalloproteinases and their endogenous inhibitors in the brain of mice subjected to ischaemic stroke. Thromb Haemost 112: 363-378, 2014

28. Xiao Q, Zhang F, Grassia G, Hu Y, Zhang Z, Xing Q, Yin X, Maddaluno M, Drung B, Schmidt B, et al: Matrix metalloproteinase- 8 promotes vascular smooth muscle cell proliferation and neointima formation. Arterioscler Thromb Vasc Biol 34: 90-98, 2014.

29. Quillard T, Araújo HA, Franck G, Tesmenitsky Y and Libby P: Matrix metalloproteinase-13 predominates over matrix metalloproteinase- 8 as the functional interstitial collagenase in mouse atheromata. Arterioscler Thromb Vasc Biol 34: 1179-1186, 2014.

30. Gupta N, Sahu A, Prabhakar A, Chatterjee T, Tyagi T, Kumari B, Khan N, Nair V, Bajaj N, Sharma M and Ashraf MZ: Activation of NLRP3 inflammasome complex potentiates venous thrombosis in response to hypoxia. Proc Natl Acad Sci USA 114: 4763-4768, 2017.

31. van Minkelen R, de Visser MC, Houwing-Duistermaat JJ, Vos HL, Bertina RM and Rosendaal FR: Haplotypes of IL1B, IL1RN, IL1R1, and IL1R2 and the risk of venous thrombosis. Arterioscler Thromb Vasc Biol 27: 1486-1491, 2007.

32. Henke PK, Varga A, De S, Deatrick CB, Eliason J, Arenberg DA, Sukheepod P, Thanaporn P, Kunkel SL, Upchurch GR Jr and Wakefield TW: Deep vein thrombosis resolution is modulated by monocyte CXCR2-mediated activity in a mouse model. Arterioscler Thromb Vasc Biol 24: 1130-1137, 2004.
33. Henke PK and Wakefield T: Thrombus resolution and vein wall injury: Dependence on chemokines and leukocytes. Thromb Res 123 (Suppl 4): S72-S78, 2009.

34. Bean CJ, Boulet SL, Ellingsen D, Trau H, Ghaji N, Hooper WC and Austin H: Increased risk of venous thromboembolism is associated with genetic variation in heme oxygenase-1 in Blacks. Thromb Res 130: 942-947, 2012.

35. Peng L, Mundada L, Stomel JM, Liu JJ, Sun J, Yet SF and Fay WP: Induction of heme oxygenase-1 expression inhibits platelet-dependent thrombosis. Antioxid Redox Signal 6: 729-735, 2004.

36. Bojic LA, Burke AC, Chhoker SS, Telford DE, Sutherland BG, Edwards JY, Sawyez CG, Tirona RG, Yin H, Pickering JG and Huff MW: Peroxisome proliferator-activated receptor $\delta$ agonist GW1516 attenuates diet-induced aortic inflammation, insulin resistance, and atherosclerosis in low-density lipoprotein receptor knockout mice. Arterioscler Thromb Vasc Biol 34: 52-60, 2014.

37. Samsoondar JP, Burke AC, Sutherland BG, Telford DE, Sawyez CG, Edwards JY, Pinkosky SL, Newton RS and Huff MW: Prevention of diet-induced metabolic dysregulation, inflammation, and atherosclerosis in $\mathrm{Ldll}^{-/}$mice by treatment with the ATP-citrate lyase inhibitor bempedoic acid. Arterioscler Thromb Vasc Biol 37: 647-656, 2017.

38. Zhu W, Buffa JA, Wang Z, Warrier M, Schugar R, Shih DM, Gupta N, Gregory JC, Org E, Fu X, et al: Flavin monooxygenase 3 , the host hepatic enzyme in the metaorganismal trimethylamine $\mathrm{N}$-oxide-generating pathway, modulates platelet responsiveness and thrombosis risk. J Thromb Haemost 16: 1857-1872, 2018.

39. Shih DM, Zhu W, Schugar RC, Meng Y, Jia X, Miikeda A, Wang Z, Zieger M, Lee R, Graham M, et al: Genetic deficiency of flavin-containing monooxygenase 3 (Fmo3) protects against thrombosis but has only a minor effect on plasma lipid levels-brief report. Arterioscler Thromb Vasc Biol 39: 1045-1054, 2019.

(i) $($ ) This work is licensed under a Creative Commons Attribution-NonCommercial-NoDerivatives 4.0 International (CC BY-NC-ND 4.0) License. 